\title{
Short-term outcome of neoadjuvant immunotherapy and chemotherapy in non-small cell lung cancer: A systematic review and meta-analysis
}

\author{
Chao Zhang, MD, ${ }^{a}$ Hui-Zhao Hong, MD, ${ }^{a}$ Yi-Long Wu, MD, ${ }^{b}$ and Wen-Zhao Zhong, MD, PhD
}

\section{ABSTRACT}

Background: Previously reported results have shown promising efficacy of neoadjuvant immunotherapy for resectable non-small cell lung cancer (NSCLC). However, no randomized control trials comparing neoadjuvant immunotherapy with chemotherapy have yet been reported. The aim of the present study was to evaluate the superiority of neoadjuvant immunotherapy compared with standard neoadjuvant chemotherapy in resectable NSCLC in terms of short-term clinical outcomes and surgical outcomes.

Methods: We searched PubMed, Embase, the Cochrane Central Register of Controlled Trials, the ClinicalTrials.gov database, Web of Science, and abstracts derived from multiple major cancer meetings up to March 1, 2020. Short-term clinical outcomes (including objective response rate [ORR], major pathologic response, and pathologic complete response [PCR]) and surgical outcomes (including surgical resection rate and Ro resection rate) were reported. Data were summarized as the estimated pooled value of each evaluated index. The risk of bias of included studies was assessed using standard methods.

Results: This systematic review and meta-analysis of 21 trials on neoadjuvant immunotherapy and neoadjuvant chemotherapy for NSCLC included 1795 patients. Patients who received Programmed death ligand 1 (PD-1/PD-L1) inhibitors (NeolO) alone $(13.3 \%$; 95\% confidence interval $[\mathrm{Cl}], 9.0 \%-19.3 \%)$ had the lowest ORR compared with those who received NeolO plus chemotherapy (CT) (62.5\%; $95 \% \mathrm{Cl}, 54.4 \%-70.0 \%$ ) or CT alone (41.6\%; $95 \% \mathrm{Cl}, 36.8 \%-46.7 \%$ ) (NeolO vs CT, $P<.001 ; \mathrm{NeolO}+\mathrm{CT}$ vs CT, $P<.001)$. Receipt of NeolO + CT (36.2\%; $95 \% \mathrm{Cl}, 19.2 \%-57.6 \%$ ) was associated with an elevated $\mathrm{pCR}$ rate compared with receipt of NeolO alone $(10.6 \% ; 95 \% \mathrm{Cl}, 6.5 \%-16.9 \% ; P<.001)$ or standard CT (7.5\%; 95\% Cl, 5.7\%-9.8\%; $P$ < .001). Neoadjuvant CT (87.2\%; $95 \% \mathrm{Cl}, 74.9 \%$ $94.0 \%$ ) was associated with a lower Ro resection rate compared with $\mathrm{NeolO}$ alone (92.7\%; $95 \% \mathrm{Cl}, 83.4 \%-97.0 \%$; $P=.360$ ) or $\mathrm{NeolO}+\mathrm{CT}$ (91.6\%; $95 \% \mathrm{Cl}, 84.3 \%$ $95.7 \% ; P=.409)$. Meta-regression showed that a higher proportion of stage III patients was correlated with decreased surgical resection and Ro resection rates, whereas no impact was observed with neoadjuvant immunotherapy.

Conclusions: Current data suggest that compared with neoadjuvant chemotherapy, immunotherapy-based regimens may provide superior pathological response along with a higher rate of complete resection. Immunotherapy combined with chemotherapy in neoadjuvant chemotherapy may be a more favorable clinical option. Further randomized controlled trials are warranted to provide long-term results of neoadjuvant immunotherapy for localized NSCLC and help guide clinical practice. (JTCVS Open 2021;8:588-607)

From the ${ }^{\mathrm{a}}$ Guangdong Lung Cancer Institute and ${ }^{\mathrm{b}}$ Department of Medical Oncology, Guangdong Lung Cancer Institute, Guangdong Provincial Key Laboratory of Translational Medicine in Lung Cancer, Guangdong Provincial People's Hospital \& Guangdong Academy of Medical Sciences, Guangzhou, China.

Supported by the National Natural Science Foundation (Grant 81872510 to W.-Z.Z.) and High-level Hospital Construction Project (grant DFJH201801 to W.-Z.Z.).

Received for publication Dec 15, 2020; accepted for publication Aug 25, 2021; available ahead of print Sept 24, 2021.

Address for reprints: Wen-Zhao Zhong, MD, PhD, Guangdong Lung Cancer Institute, Guangdong Provincial People's Hospital \& Guangdong Academy of Medical

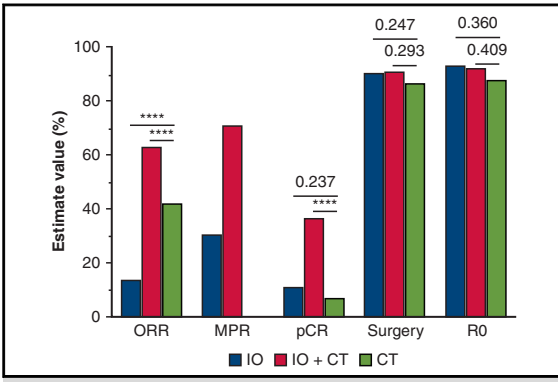

Meta-analysis comparing neoadjuvant immunotherapy and chemotherapy.

\section{CENTRAL MESSAGE}

The pooled data suggest a high pathological response rate and higher surgical resection rate for immunotherapy-based neoadjuvant treatment compared with standard chemotherapy for treating resectable non-small cell lung cancer.

\section{PERSPECTIVE}

Standard neoadjuvant therapy remains the standard of care chemotherapy for non-small cell lung cancer despite the minor improvements in 5-year overall survival rate and high treatment-related adverse events. Neoadjuvant immunotherapy, especially when combined with chemotherapy, could provide a relatively high pathological response rate and improve surgical outcome.
Sciences, Guangzhou, Zhongshan Er Rd No.106, Guangdong 510080, China (E-mail: syzhongwenzhao@scut.edu.cn).

JTCVS Open 2021;8:588-607 2666-2736

Copyright (C) 2021 The Author(s). Published by Elsevier Inc. on behalf of The American Association for Thoracic Surgery. This is an open access article under the CC BY-NC-ND license (http://creativecommons.org/licenses/by-nc-nd/4.0/). https://doi.org/10.1016/j.xjon.2021.08.036 

Abbreviations and Acronyms
$\mathrm{CI}=$ confidence interval
$\mathrm{CT}=$ chemotherapy
IO = immunotherapy
MPR = major pathologic response
Neo = neoadjuvant
NSCLC $=$ non-small cell lung cancer
ORR = objective response rate
$\mathrm{pCR}=$ pathologic complete response
PDL-1 = programmed death ligand 1

Recent advances in treatment involving tyrosine kinase inhibitors and immune checkpoint inhibitors have shifted the treatment paradigm for late-stage cancer, with significantly prolonged survival along with lower toxicity. ${ }^{1}$ Unlike in advanced disease, in which surgery may have a limited role, the standard of care for early-stage disease remains definitive local treatment, such as surgery and radiotherapy. ${ }^{2}$ However, patients who undergo radical resection alone could still be at high risk for disease recurrence. ${ }^{3}$ Previous trials have suggested perioperative chemotherapy (CT) as a recommended treatment modality for earlystage non-small cell lung cancer (NSCLC) ${ }^{4}$; however, despite its superiority over surgery alone, only a $\sim 5 \%$ increase in 5-year survival rate has been observed, with considerable treatment-related toxicity. ${ }^{5-7}$ Therefore, superior therapeutic modalities are urgently needed.

Given the long-term benefit of immunotherapy in advanced cancer, trials evaluating the clinical outcomes of neoadjuvant immunotherapy (NeoIO) have shown encouraging results, with high rates of major pathologic response (MPR). ${ }^{8,9}$ It should be noted that most of these trials were single-arm studies with no comparisons with traditional CT. Moreover, a lack of mature prognostic data in these trials makes it difficult to determine the long-term benefit of NeoIO. Indeed, a previous study indicated that both radiologic response and pathologic regression can help predict survival in patients with early-stage NSCLC, suggesting that these 2 clinical outcomes can serve as surrogate endpoints. ${ }^{10}$ Considering that no data from randomized controlled trials on NeoIO and CT have been reported to date, we performed a metaanalysis of studies on NeoIO and CT in patients with early-stage NSCLC to better define more optimal treatment modalities than NeoCT.

\section{METHODS}

This study is reported following the Preferred Reporting Items for Systematic Reviews and Meta-Analyses (PRISMA) statement, excluding the protocol and registration number. A flow diagram of the study is shown in Figure 1.

\section{Data Source and Searches}

We searched PubMed, Embase, the Cochrane Central Register of Controlled Trials, the ClinicalTrials.gov database, and Web of Science to identify all relevant English language articles published up to March 1, 2020, using combined search terms including "neoadjuvant immunotherapy" and "NSCLC." The specific search terms for NeoIO and CT are provided in Online Data Supplement. We also searched the reference lists of included studies. Given the very limited number of neoadjuvant trials published to date, we also included recently reported data, especially those reported at relevant meetings after March 1, 2020. To avoid missing any unpublished updated data, we also searched the databases of several major cancer-related international conferences (ie, World Conference on Lung Cancer, European Society of Medical Oncology, American Society of Clinical Oncology, European Lung Cancer Conference, and American Association for Cancer Research) from 2010 to 2019 for relevant content using the same search terms noted above. If duplicate studies were found from the same cohort that offered similar outcome measures, we included the studies reporting the most relevant data. In addition, referenced articles for the relevant studies were checked in cases of missing data.

\section{Study Selection}

For all first included studies, only phase II/III prospective trials on NeoCT and phase I/II prospective trials on NeoIO were included for further assessment. The study selection process is summarized in Figure 1. Studies enrolled for sequential meta-analysis were trials that enrolled patients whose tumor was pathologically confirmed as stage I-III NSCLC regardless of the oncogene mutation. NeoCT that involved treatment beyond chemotherapy, including radiotherapy in a neoadjuvant setting, were excluded. For NeoIO trials, we allowed those combination modalities with CT, owing to the limited reported data. Trials included in the metaanalysis had to include the objective response rate (ORR) of neoadjuvant treatment before surgery and at least 1 of the following clinical outcomes: MPR (viable tumor cells $<10 \%$ ), pathologic complete response (pCR), and complete reports of adverse events (optional for NeoIO, as most of those trials had not been officially published).

There was no restriction on specific regimens for all patients or dependence on histology in the NeoCT trials. In addition, cohorts with NeoCT plus radiotherapy were excluded. Because we could not compare prognostic data between 2 treatment modalities owing to immature data on $\mathrm{NeoIO}$, no requirement for long-term follow-up was needed for the included trials. Two reviewers (Z.C. and H.H.Z.) independently screened all titles and abstracts that met the inclusion criteria. For those titles and abstracts that met the foregoing criteria, the same reviewers retrieved available corresponding articles (optional for NeoIO) to assess them for final inclusion in the meta-analysis. Any disagreements were resolved by consensus.

\section{Data Extraction and Risk of Bias Assessment}

Two reviewers (Z.C. and H.H.Z.) performed independent double data extraction. Detailed data on trials (eg, intervention type, study title, first author, NCT number, number of patients within the neoadjuvant treatment group, baseline characteristics of the enrolled patients), treatment regimens as well as duration, and short-term clinical outcomes (eg, radiologic response, pathologic regression) were extracted for consideration in subsequent analyses. Because survival data on the NeoIO cohort were too limited and immature to calculate, we decided to not compare the treatment modalities in terms of prognosis. The information was obtained from published data or unpublished data or calculated using the raw data.

Considering the disparity among trials measuring different clinical outcomes, we unified all evaluated outcomes. ORR was defined as the number of patients with partial or complete response divided by the number of patients who received at least 1 dose of neoadjuvant therapy. MPR was 
Neoadjuvant immunotherapy cohorts

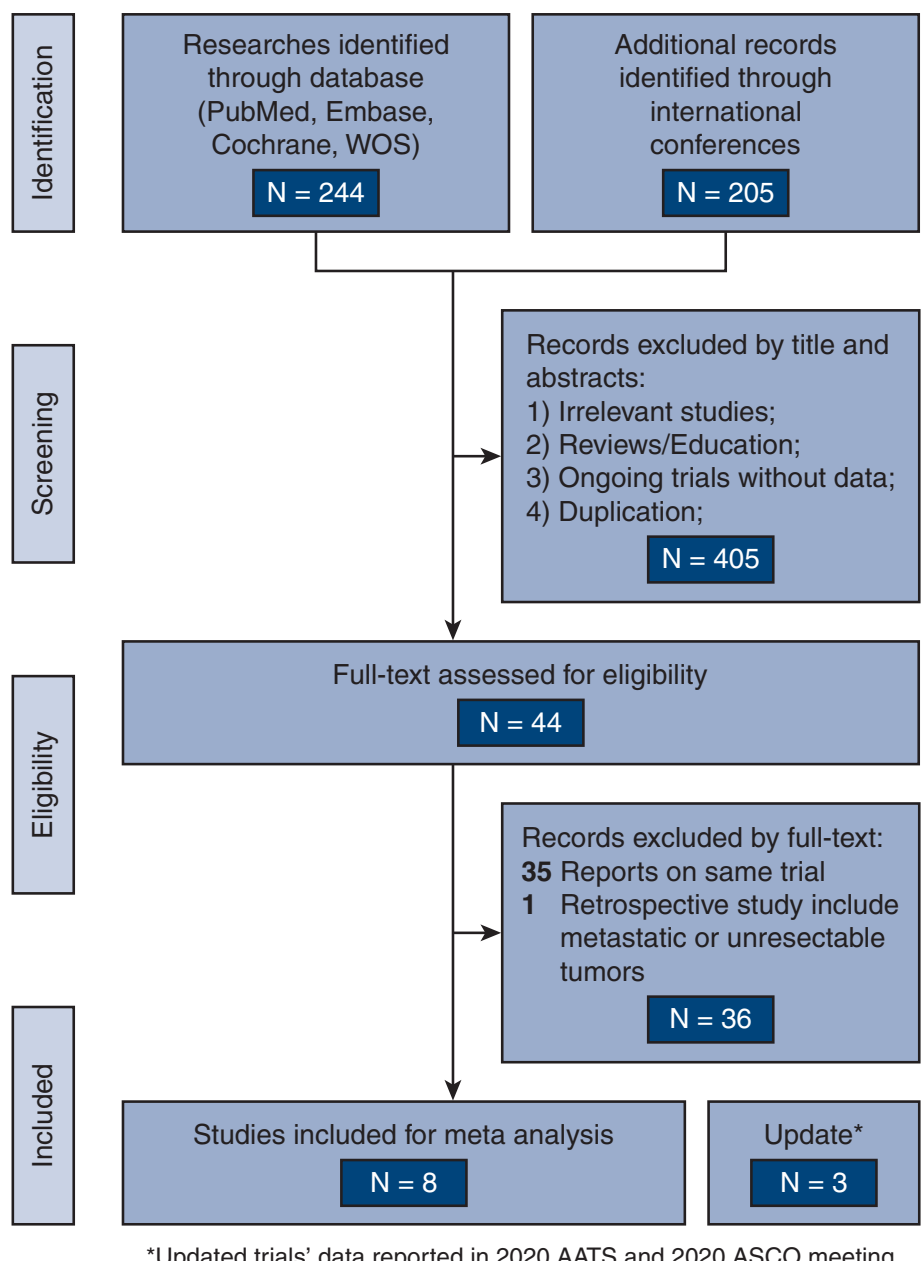

Neoadjuvant chemotherapy cohorts

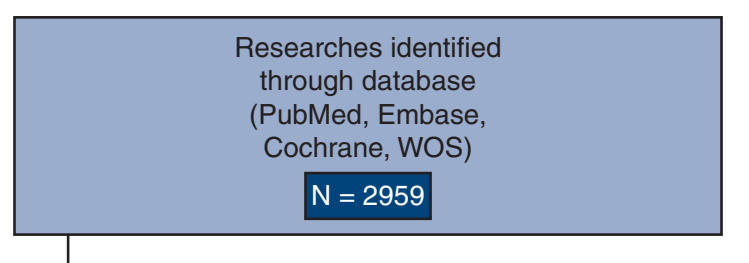

Records excluded by title and

abstracts:

1) Irrelevant studies;

2) Reviews/Education;

3) Ongoing trials without data;

4) Duplication;

$N=2886$

Full-text assessed for eligibility

$\mathrm{N}=73$

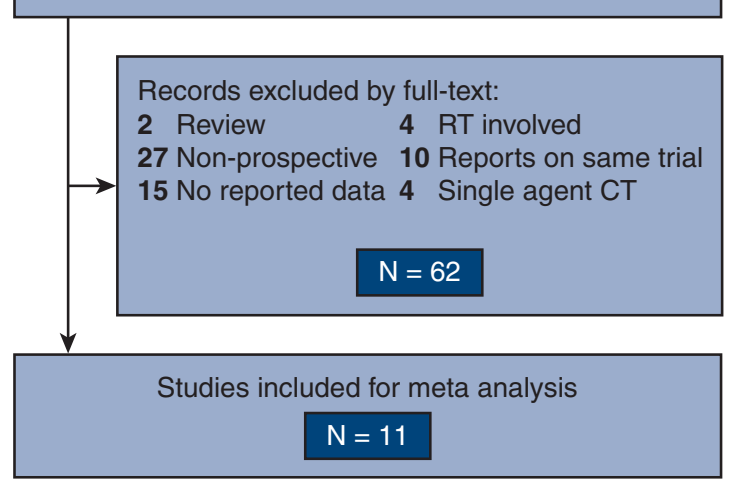

${ }^{\#} \mathrm{RT}$, radiotherapy; CT, chemotherapy

FIGURE 1. Summary of the 4 major processes for study selection, including the number of initial collected studies, excluded studies, and enrolled studies. Titles and abstracts that met the inclusion criteria were further assessed in a full text review for inclusion in the meta-analysis. Novel data reported at the 2020 American Association for Thoracic Surgery (AATS) and 2020 American Society of Clinical Oncology (ASCO) meetings were included. WOS, World of Science; $C T$, chemotherapy.

defined as the number of patients with MPR divided by the number of patients who had evaluable resected tissues (for NeoIO only, because no relevant data were reported for NeoCT). pCR was defined as the number of patients with $\mathrm{pCR}$ divided by the number of patients who had evaluable resected tissues. Surgical resection rate was defined as the number of patients who successfully underwent surgery divided by the number of patients who received at least 1 dose of neoadjuvant therapy. R0 resection rate was defined as the number of patients who underwent complete resection with negative margins divided by the number of patients in whom surgery was successfully performed.

The same reviewers (Z.C. and H.H.Z.) independently assessed the risk of each study. Specifically, we used the Cochrane risk of bias tool to evaluate studies of NeoCT in terms of the following domains: random sequence generation, allocation concealment, blinding of participants and personnel, blinding of outcome assessment, incomplete outcome data, selective outcome reporting, and other sources of bias. These aspects were scored as low, high, or unclear risk of bias. For NeoIO trials, we applied the Methodologic Index for Nonrandomized Studies (MINORS) to assess the bias of these trials.

\section{Data Synthesis and Analysis}

Statistical analysis was performed using R version 3.6.1 (R Foundation for Statistical Computing, Vienna, Austria), Prism version 7.00 (GraphPad
Software, La Jolla, Calif) and SPSS Statistics for Windows, version 20.0 (IBM, Armonk, NY). The R META package was used for the metaanalysis and meta-regression. For proportions, the R function METAPROP, which uses the log-odds (ie, logit transformation) was applied. All included studies were combined in a descriptive synthesis, comparing NeoIO and CT in terms of multiple short-term clinical outcomes. The meta-analysis was performed based on 3 major groups: neoadjuvant programmed death ligand 1 (PD-1/PD-L1) inhibitors alone (NeolO), PD-1/PD-L1 inhibitors plus chemotherapy (NeoIO + CT), and neoadjuvant chemotherapy (NeoCT). Each group was evaluated based on several short-term clinical outcomes, including ORR, MPR, pCR, and surgical outcome (including surgical resection rate and R0 resection rate). We assessed heterogeneity using $I^{2}$ and $\tau^{2}$ values and calculated the estimates for the aforementioned clinical outcomes together with their $95 \%$ confidence intervals (CIs). Determination of which model to use to calculate the estimated values was made on both the authors' assessment and statistical heterogeneity ${ }^{11}$ Funnel plots were created to evaluate the publication bias of each included study. ${ }^{12}$ Egger's test and Begg's test were used the check for publication bias. In consideration of the limited trials included for this study, meta-regression was applied to compare chemotherapy-based and immunotherapy-based modalities regarding short-term outcomes and to evaluate the moderating variables that could impact the results. All reported $P$ values were 2 -sided, and statistical significance was defined as $P<.05$. 


\section{RESULTS}

\section{Systematic Review and Characteristics}

Using the predefined search terms, we identified 449 relevant records for PD-1/PD-L1-based NeoIO and 2959 relevant records for NeoCT. After the manual screening and eligibility assessment, 10 studies $^{8,9,13-16}$ for NeoIO and 11 studies ${ }^{17-27}$ for NeoCT were included for further analysis, including 400 and 1395 patients, respectively. Specifically, the included NeoIO trials included 6 PD-1/PD-L1-based single-agent trials and 5 immunotherapy combination cohorts; the NEOSTAR trial contained both single-agent and dual-agent immunotherapy cohorts. All included NeoCT trials used cisplatin- or carboplatin-based chemotherapy as neoadjuvant regimens. Detailed characteristics of each study are summarized in Table 1.

\section{Short-Term Efficacy in Neoadjuvant Trials}

We first evaluated the available short-term outcomes for both neoadjuvant immunotherapy and chemotherapy according to the 3 major groups: $\mathrm{NeoIO}$, NeoIO $+\mathrm{CT}$, and NeoCT. Nine studies on NeoIO and 11 studies on NeoCT were eligible for ORR estimation, and 8 studies on NeoIO and 7 studies on NeoCT were eligible for pCR estimation. All forest plots were generated to estimate ORR, MPR, and pCR, based on a fixed- or random-effect model.

Regarding ORR, NeoIO alone showed an inferior radiologic response compared with traditional NeoCT $(13.3 \%$ [95\% CI, 9.0\%-19.3\%] vs $41.6 \%$ [95\% CI, 36.8\%$46.7 \%])$. NeoIO + CT yielded the highest ORR among the 3 groups $(62.5 \%$; $95 \%$ CI, $54.4 \%-70.0 \%)$. In terms of pCR, NeoCT showed similar pathologic regression as NeoIO alone (7.5\% [95\% CI, 5.7\%-9.8\%] vs $10.6 \%$ [95\% CI, 6.5\%-16.9\%]), whereas NeoIO + CT showed superior pathological regression $(36.2 \% ; 95 \%$ CI, 19.2\%-57.6\%) (Figure 2). Because of the limited MPR data presented in the NeoCT trials, we compared this clinical outcome only in the NeoIO and NeoIO + CT groups. As expected, MPR was significantly higher in the $\mathrm{NeoIO}+\mathrm{CT}$ group $(70.5 \%[95 \%$ CI, $54.9 \%-82.4 \%]$ vs $30.0 \%$ [95\% CI, 20.5\%-41.7\%]) (Figure E1). In particular, we observed favorable short-term clinical outcomes with PD-1 inhibitors, yet the limited included trials on PD-L1 inhibitors might have underpowered the statistical evaluation.

\section{Outcome for Sequential Surgery}

There is a potential risk of patients receiving systemic treatment, such as targeted therapy or immunotherapy, before surgery being unavailable for sequential surgical resection owing to severe toxicity or disease progression. Ineligibility for surgery would impair these patients' prognosis because these are all potentially resectable diseases.
Moreover, those patients who undergo surgery also may undergo non-R0 resection owing to heterogeneous responses to the disease. Thus, we assessed surgical rates and R0 resection rates after the different neoadjuvant modalities to evaluate the outcomes of sequential surgery in the patients.

All 3 therapeutic regimens achieved a satisfactory surgical rate after neoadjuvant treatment, with $89.8 \%$ (95\% CI, $84.9 \%-93.3 \%)$ for NeoIO alone, $90.3 \%(95 \% \mathrm{CI}$, $84.1 \%-94.2 \%)$ for NeoIO + CT, and $85.9 \%(95 \% \mathrm{CI}$, $77.9 \%-91.3 \%$ ) for NeoCT. For R0 resection rate, NeoCT showed relatively worse performance $(87.2 \% ; 95 \%$ CI, 74.9\%-94.0\%) compared with NeoIO (92.7\%; $95 \%$ CI, $83.5 \%-96.9 \%)$ and NeoIO + CT $(91.6 \% ; 95 \% \mathrm{CI}$, $84.3 \%-95.7 \%$ ) (Figure 3).

\section{Comparisons Through Meta-Regression}

Comparison involving ORR, pCR, surgical rate, and R0 resection rate were conducted via meta-regression. Regarding the short-term clinical outcomes, NeoIO + CT showed superior efficacy of the 3 groups (Figure 4). Regarding the proportion with stage III disease and lung squamous cell carcinoma, we found significant correlation between the proportions of patients with stage III disease and patients with ORR/MPR. However, when this index was integrated with the treatment modalities (NeoIO alone and NeoIO + CT), only the treatment modalities could significantly affect short-term clinical outcomes (Figure E2). Through meta-regression, we found that higher proportion of stage III patients could be correlated with both an inferior surgical resection rate and an inferior R0 resection rate through NeoCT, whereas no such correlation was found with $\mathrm{NeoIO}$ and $\mathrm{NeoIO}+\mathrm{CT}$, suggesting a potential advantage of neoadjuvant immunotherapy for surgical resection (Figure E3). Overall, these results support $\mathrm{NeoIO}+\mathrm{CT}$ as possibly the most ideal option for resectable NSCLC.

\section{Study Heterogeneity and Risk of Bias}

The heterogeneity among studies was quantified using $I^{2}$ and $\tau^{2}$ values, and most of the analyses revealed either mild or intermediate heterogeneity. Funnel plots showed several outliers but were relatively centralized overall, suggesting that there was limited publication bias in the included trials (Figures E4-E6).

A risk of bias assessment for each included study was performed using the Cochrane risk of bias tool and MINORS for NeoCT and NeoIO trials, respectively. Specifically, for NeoCT trials, because all the studies compared NeoCT and surgery alone without the use of placebo, information on blinding was unavailable. However, because this is not a direct comparison, such bias might not significantly 
TABLE 1. Summary of included neoadjuvant immunotherapy and chemotherapy trials

\begin{tabular}{|c|c|c|c|c|c|c|c|c|c|}
\hline Trial & $\mathbf{N}$ & $\begin{array}{l}\text { Males, } \\
\text { n (\%) }\end{array}$ & $\begin{array}{c}\text { Age, } \mathbf{y}, \\
\text { median } \\
(\text { range }) / \\
\text { mean } \pm \text { SD }\end{array}$ & $\begin{array}{c}\text { Histology } \\
\text { (SQC), n (\%) }\end{array}$ & $\begin{array}{c}\text { Stage } \\
(\text { TNM) }\end{array}$ & $\begin{array}{l}\text { Stage III } \\
\text { proportion, } \\
\text { n }(\%)\end{array}$ & $\begin{array}{l}\text { Neoadjuvant } \\
\text { regimen }\end{array}$ & $\begin{array}{c}\text { Treatmen } \\
\text { cycles }\end{array}$ & $\begin{array}{r}\text { It Primary } \\
\text { endpoint }\end{array}$ \\
\hline \multicolumn{10}{|c|}{ Immunotherapy-based neoadjuvant treatment } \\
\hline Checkmate $159^{8}$ & 21 & $10(47.6)$ & $67(55-84)$ & $6(28.6)$ & I-IIIA (7th) & $7(33.3)$ & Nivolumab & 2 & $\begin{array}{l}\text { Safety/ } \\
\text { outcome }\end{array}$ \\
\hline $\mathrm{LCMC}^{13}$ & 101 & $47(46.5)$ & $65(37-83)$ & $35(34.7) \quad 1$ & IB-IIIB (8th) & $46(45.5)$ & Atezolizumab & 2 & MPR \\
\hline TOP1501* & 30 & $16(53.3)$ & $72(47-81)$ & $17(56.7) \quad \mathrm{I}$ & IB-IIIA (7th) & $8(26.7)$ & Pembrolizumab & 2 & $\begin{array}{l}\text { Safety/ } \\
\text { outcome }\end{array}$ \\
\hline MK3457-233 $3^{15}$ & 13 & $6(46.2)$ & $66(\mathrm{NR})$ & $6(46.2)$ & I-IIB (8th) & 0 & Pembrolizumab & 2 & Safety \\
\hline Sintilimab $^{16}$ & 40 & $33(82.5)$ & $62(47-70)$ & $33(82.5)$ & I-IIIB (8th) & $18(45.0)$ & Sintilimab & 2 & Safety \\
\hline NEOSTAR (single) $\dagger$ & 23 & $15(65.2)$ & $66.1 \pm 8.5$ & $10(43.5)$ & I-IIIA (7th) & $5(21.7)$ & Nivolumab & 3 & MPR \\
\hline NEOSTAR (comb) $\dagger$ & 21 & 13 (61.9) & $65.0 \pm 8.3$ & $7(33.3)$ & I-IIIA (7th) & $4(19.0)$ & Nivolumab + ipilimumab & 3 & MPR \\
\hline MAC $^{9}$ & 30 & $15(50.0)$ & $67(62-74)$ & $12(40.0)$ & II-IIIA (7th) & $23(76.7)$ & $\begin{array}{c}\text { Atezolizumab }+ \text { paclitaxel/ } \\
\text { carboplatin }\end{array}$ & 4 & MPR \\
\hline NADIM $^{14}$ & 46 & $34(73.9)$ & $63(41-77)$ & NR & IIIA (7th) & $46(100.0)$ & $\begin{array}{c}\text { Nivolumab + paclitaxel/ } \\
\text { carboplatin }\end{array}$ & 3 & 2-y PFS \\
\hline SAKK $16 / 14 \dagger$ & 67 & $35(52.2)$ & $61(41-74)$ & $22(32.8)$ & IIIA (7th) & $67(100.0)$ & $\begin{array}{c}\text { Durvalumab }+ \text { docetaxel/ } \\
\text { cisplatin }\end{array}$ & 5 & 1-y EFS \\
\hline NCT03366766† & 13 & $8(61.5)$ & $69(49-80)$ & $9(69.2)$ & IB-IIIA (7th) & $7(53.8)$ & $\begin{array}{c}\text { Nivolumab }+ \text { histology- } \\
\text { based chemotherapy }\end{array}$ & 3 & MPR \\
\hline IO-based regimen & 405 & $232(57.3)$ & - & $157(38.8)$ & - & $231(57.0)$ & - & $2-5$ & - \\
\hline \multicolumn{10}{|c|}{ Chemotherapy-based neoadjuvant treatment } \\
\hline JCOG9209 ${ }^{17}$ & 31 & $20(64.5)$ & $59(32-74)$ & $7(22.6)$ & IIIA & $31(100.0)$ & Cisplatin/vindesine & 3 & NR \\
\hline Chen et al. $(2013)^{24}$ & 169 & $132(78.1)$ & $61(34-75)$ & $79(46.7)$ & I-IIIA & $66(39.1)$ & $\begin{array}{l}\text { Mitomycin/cisplatin/ } \\
\text { vindesine }\end{array}$ & 4 & OS/PFS \\
\hline CHEST $^{23}$ & 129 & $100(77.5)$ & $60.6(37.6-76.3)$ & $48(37.2)$ & IA-IIIA & $6(4.7)$ & Gemcitabine/cisplatin & 3 & PFS \\
\hline $\mathrm{CSLC} 0501^{27}$ & 97 & 79 (81.4) & $58(26-75)$ & NR & IB-IIIA & $29(29.9)$ & Docetaxel/carboplatin & 3 & 3-y DFS \\
\hline $\begin{array}{l}\text { MRCLU22/NVALT2/ } \\
\text { EORTC08012 }\end{array}$ & 258 & $186(72.1)$ & $62(37-77)$ & $131(50.8)$ & IA-IIIB & $21(8.1)$ & $\begin{array}{l}\text { MVP/MIC/NP/paclitaxel/ } \\
\text { carboplatin/gemcitabine/ } \\
\text { cisplatin/docetaxel/ } \\
\text { carboplatin }\end{array}$ & 3 & OS \\
\hline NACTH III ${ }^{22}$ & 199 & 175 (87.9) & $65(35-80)$ & $107(53.8)$ & IA-IIB & 0 & Paclitaxel/carboplatin & 3 & DFS \\
\hline SAKK $16 / 00^{25}$ & 115 & $77(67.0)$ & $59(30-74)$ & $36(31.3)$ & IIIA & $115(100.0)$ & Cisplatin/docetaxel & 3 & EFS \\
\hline $\mathrm{GLCCG}^{21}$ & 260 & $215(82.7)$ & $59(35-69)$ & $148(56.9)$ & IIIA-B & $260(100.0)$ & Cisplatin/etoposide & 3 & PFS \\
\hline $\operatorname{GINEST}(\mathrm{GC})^{19}$ & 12 & $5(41.7)$ & $61.5(42-83)$ & $5(41.7)$ & IA-II & 0 & Gemcitabine/cisplatin & 3 & $\mathrm{pCR}$ \\
\hline $\operatorname{GINEST}(\mathrm{GP})^{19}$ & 35 & $20(57.1)$ & $63(33-79)$ & 17 (48.6) & IA-II & 0 & Gemcitabine/paclitaxel & 3 & pCR \\
\hline $\operatorname{GINEST}(\mathrm{GCb})^{19}$ & 40 & $21(52.5)$ & $63.5(36-82)$ & $12(30.0)$ & IA-II & 0 & Gemcitabine/carboplatin & 3 & pCR \\
\hline Zhao et al. $(2016)^{26}$ & 10 & $9(90.0)$ & $58(36-63)$ & $3(30.0)$ & IIIA & $10(100.0)$ & Vinorelbine/cisplatin & 2 & $\begin{array}{c}\mathrm{RR} / \mathrm{CBR} / \\
\mathrm{TRR}\end{array}$ \\
\hline $\mathrm{JCOG} 0204^{20}$ & 40 & NR & NR & $10(25.0)$ & IB-II & 0 & Cisplatin/docetaxel & 2 & 1-y DFS \\
\hline $\begin{array}{l}\text { Chemotherapy-based } \\
\text { regimen }\end{array}$ & 1395 & $1039(74.5)$ & - & $603(43.2)$ & - & $538(38.6)$ & - & $2-4$ & - \\
\hline
\end{tabular}

$S D$, Stable disease; $S Q C$, squamous cell carcinoma; $M P R$, major pathologic response; $P F S$, progression-free survival; $E F S$, event-free survival; $N R$, not reported; $O S$, overall survival; DFS, disease-free survival; $M V P$, Mitomycin/Vinblastine/Cisplatin; $M I C$, Mitomycin/Ifosfamide/Cisplatin; $N P$, Cisplatin/Vinorelbine; $p C R$, pathologic complete response; $R R$, response rate; $C B R$, clinical benefit rates; $T R R$, tumor regression rate. *Study reported at the 2020 AATS meeting. $\dagger$ Studies reported at the 2020 ASCO meeting.

influence the results. In addition, by integrating metaregression in subgroup analysis for NeoIO, we found a significant discrepancy only between NeoIO alone and $\mathrm{NeoIO}+\mathrm{CT}$, which we did not combine for subsequent analyses. Indeed, most of the other aspects had a low risk of bias. For NeoIO, all included studies showed no significant bias (Figure E7).

\section{DISCUSSION}

In this systematic review and meta-analysis, we have comprehensively summarized the short-term efficacy and surgical outcomes of multiple reported neoadjuvant immunotherapy trials published to date. Subsequently, we performed a meta-analysis of these neoadjuvant immunotherapy trials with neoadjuvant chemotherapy cohorts derived from 


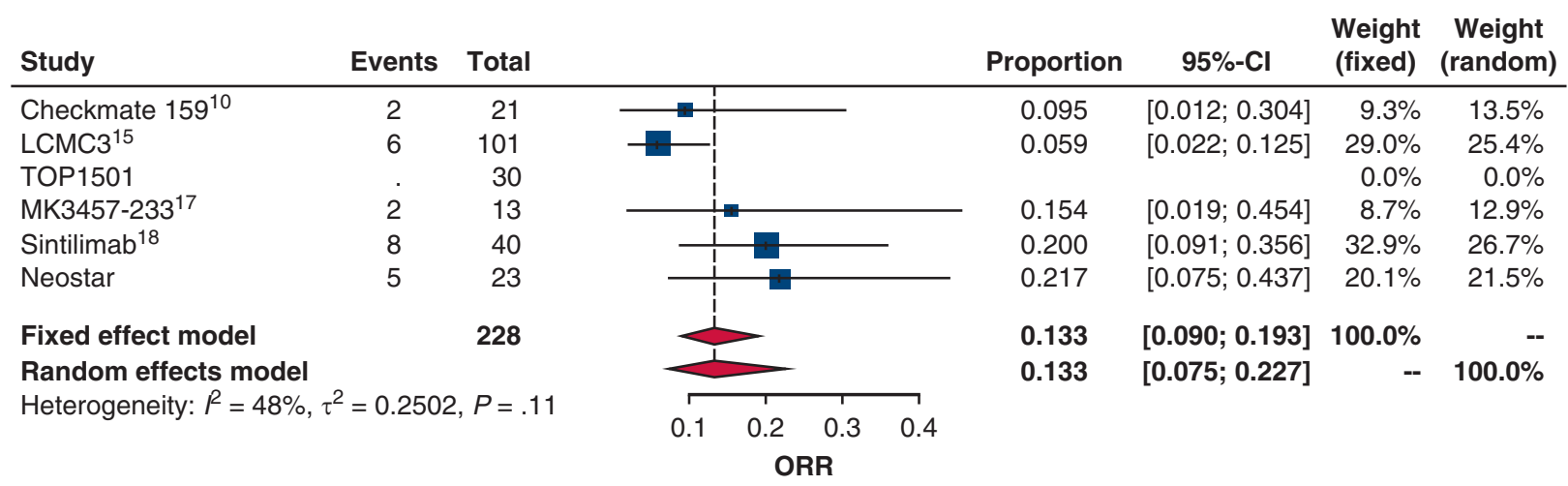

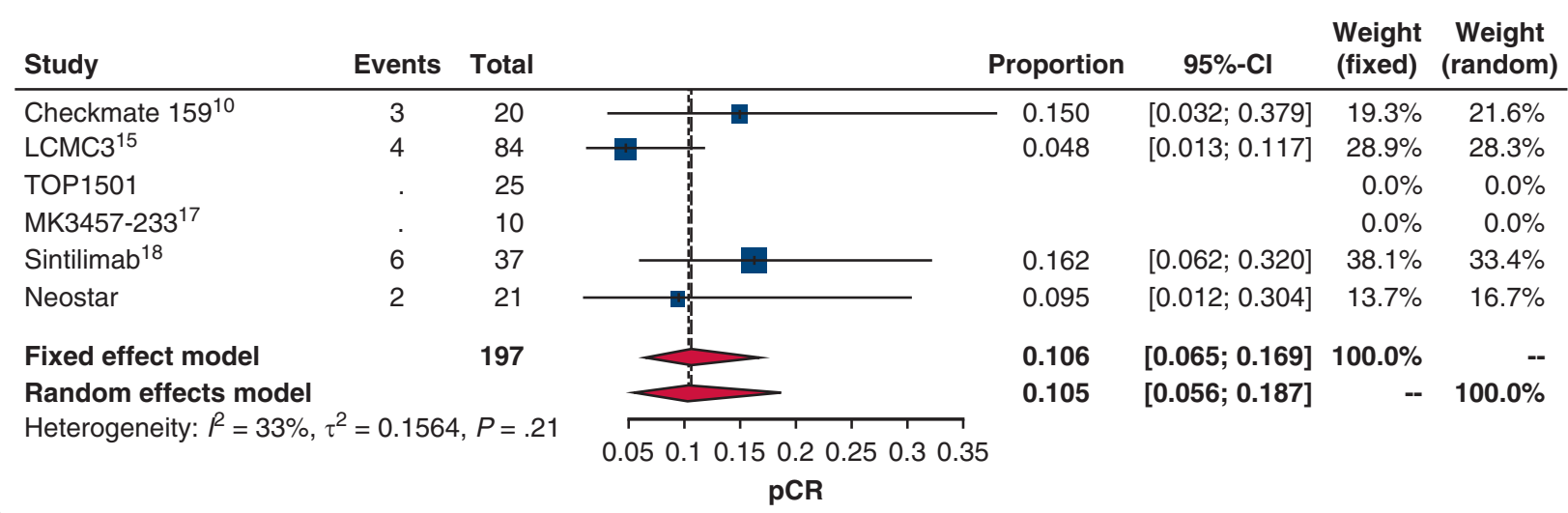

A

\begin{tabular}{|c|c|c|c|c|c|c|}
\hline Study & Events & Total & Proportion & $95 \%-\mathrm{Cl}$ & $\begin{array}{l}\text { Weight } \\
\text { (fixed) }\end{array}$ & $\begin{array}{c}\text { Weight } \\
\text { (random) }\end{array}$ \\
\hline $\mathrm{MAC}^{11}$ & 19 & 30 & 0.633 & {$[0.439 ; 0.801]$} & $20.4 \%$ & $23.1 \%$ \\
\hline NADIM $^{16}$ & 34 & 46 & 0.739 & {$[0.589 ; 0.857]$} & $26.0 \%$ & $27.2 \%$ \\
\hline SAKK16/14 & 36 & 62 & 0.581 & {$[0.448 ; 0.705]$} & $44.2 \%$ & $37.0 \%$ \\
\hline NCT03366766 & 6 & 13 & 0.462 & {$[0.192 ; 0.749]$} & $9.5 \%$ & $12.8 \%$ \\
\hline $\begin{array}{l}\text { Fixed effect model } \\
\text { Random effects model }\end{array}$ & & 151 & $\begin{array}{l}0.625 \\
0.625\end{array}$ & $\begin{array}{l}{[0.544 ; 0.700]} \\
{[0.521 ; 0.718]}\end{array}$ & $\begin{array}{r}100.0 \% \\
--\end{array}$ & $100.0 \%$ \\
\hline
\end{tabular}

Heterogeneity: $P=33 \%, \tau^{2}=0.0620, P=.22$

$\begin{array}{lllllll}0.2 & 0.3 & 0.4 & 0.5 & 0.6 & 0.7 & 0.8\end{array}$

ORR

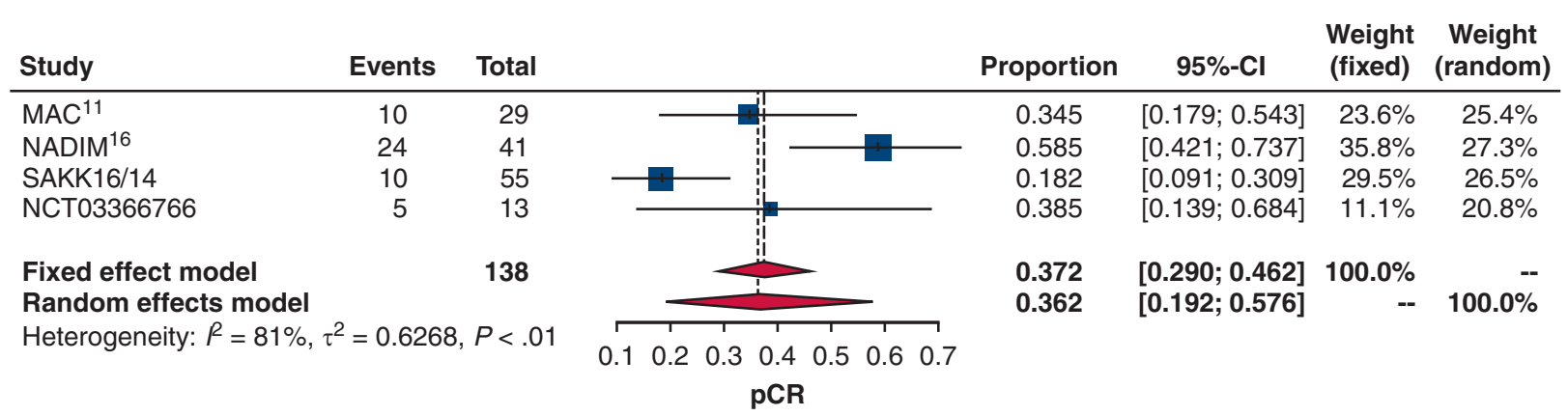

B

FIGURE 2. Estimates of objective response rate $(O R R)$ and $(p C R)$ among different treatment modalities. Estimated values for short-term clinical outcomes in 3 different treatment cohorts are shown. Vertical lines in the forest plots indicates estimates of ORR or pCR in different statistical models. The weight of each individual study in the different statistical models is provided on the right side of forest plots. A, Neoadjuvant immunotherapy (NeoIO) alone. B, NeoIO plus chemotherapy (CT). C, Neoadjuvant CT alone. $C I$, Confidence interval. 


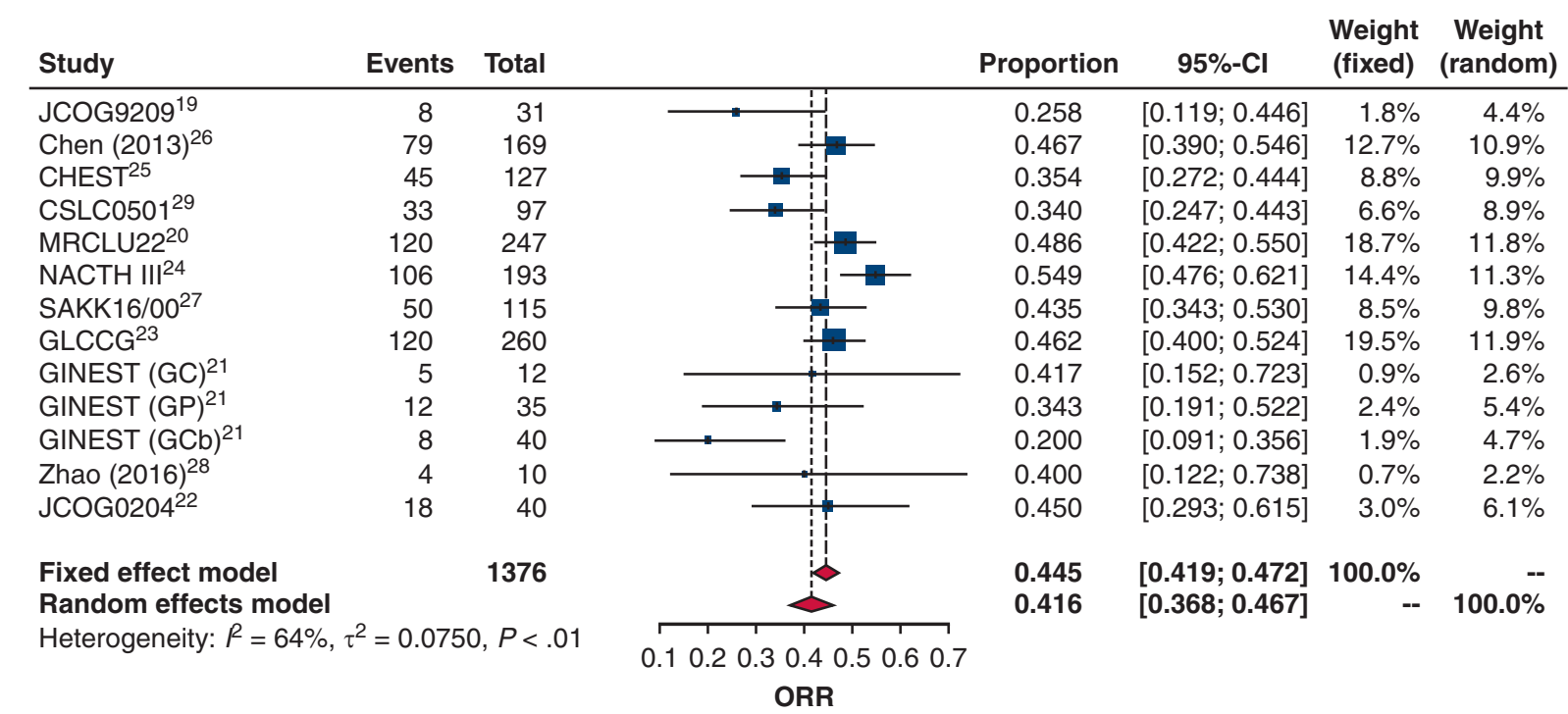

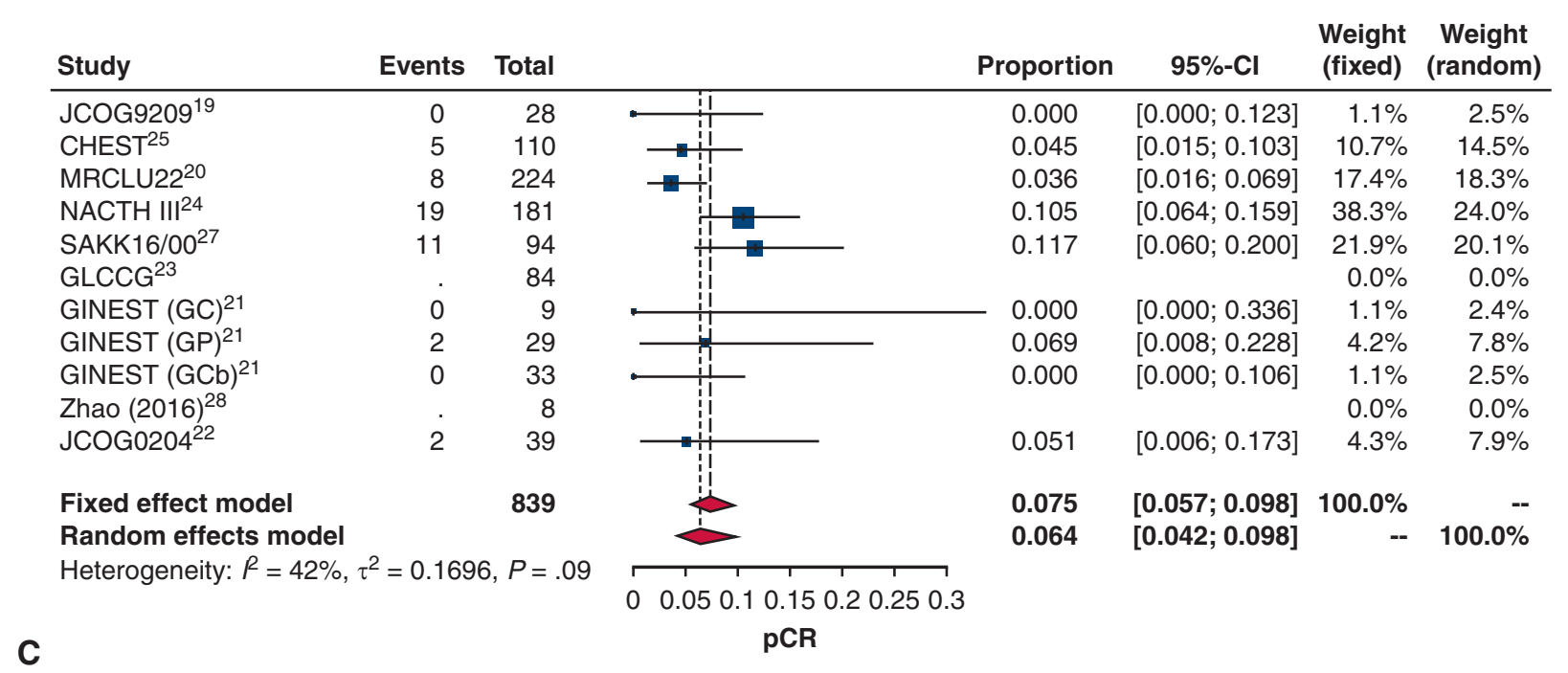

FIGURE 2. Continued.

randomized trials that aimed to address the question of whether neoadjuvant immunotherapy with or without chemotherapy is superior to neoadjuvant chemotherapy alone (Figure 5). Recently released data from Checkmate816 clarified the clinical efficacy of neoadjuvant immunotherapy plus chemotherapy. Similarly, in our study, we found that neoadjuvant immunotherapy plus chemotherapy can provide both superior radiologic and pathologic responses along with improved surgical outcomes, and likely will become the standard of care pending long-term results.

For decades, neoadjuvant chemotherapy has been maintained as the major regimen for early-stage NSCLC based on multiple prospective trials indicating a 5-year survival benefit with additional chemotherapy prior to surgery. The aim of neoadjuvant treatment is to eradicate micrometastatic lesions, increase the complete surgical resection rate, prolong the time to disease recurrence, and improve overall survival. ${ }^{28}$ However, as cytotoxic agents, chemotherapy inevitably causes adverse events, including intestinal reactions and myelosuppression, leading to patients' unwillingness to undergo continuous treatment. ${ }^{29}$ Multiple early-phase trials of neoadjuvant immunotherapy for early-stage NSCLC have been initiated, but few have reported detailed data. Our results suggest that neoadjuvant immunotherapy alone can improve the pathologic response to tumors but not the radiologic response compared with chemotherapy. One possible explanation for this is that radiologic response might not fully reflect pathologic regression in consideration of infiltrating immune cells and tissue fibrosis. ${ }^{10,16}$ In addition, trials of single-agent immunotherapy for advanced NSCLC showing comparable ORR with doublet chemotherapy 


\begin{tabular}{|c|c|c|c|c|c|c|c|}
\hline Study & Events & Total & & Proportion & $95 \%-\mathrm{Cl}$ & $\begin{array}{l}\text { Weight } \\
\text { (fixed) }\end{array}$ & $\begin{array}{l}\text { Weight } \\
\text { (random) }\end{array}$ \\
\hline Checkmate $159^{10}$ & 21 & 21 & 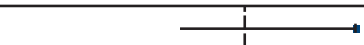 & 1.000 & {$[0.839 ; 1.000]$} & $2.6 \%$ & $2.6 \%$ \\
\hline LCMC3 $^{15}$ & 90 & 101 & & 0.891 & {$[0.813 ; 0.944]$} & $52.5 \%$ & $52.5 \%$ \\
\hline TOP1501 & 25 & 30 & + & 0.833 & {$[0.653 ; 0.944]$} & $22.3 \%$ & $22.3 \%$ \\
\hline MK3457-233 ${ }^{17}$ & 13 & 13 & & 1.000 & {$[0.753 ; 1.000]$} & $2.6 \%$ & $2.6 \%$ \\
\hline Sintilimab $^{18}$ & 37 & 40 & 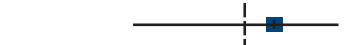 & 0.925 & {$[0.796 ; 0.984]$} & $14.9 \%$ & $14.9 \%$ \\
\hline Neostar & 22 & 23 & & 0.957 & {$[0.781 ; 0.999]$} & $5.1 \%$ & $5.1 \%$ \\
\hline \multirow{2}{*}{\multicolumn{2}{|c|}{$\begin{array}{l}\text { Fixed effect model } \\
\text { Random effects model }\end{array}$}} & 228 & & 0.898 & {$[0.849 ; 0.933]$} & $100.0 \%$ & -- \\
\hline & & & & 0.898 & {$[0.849 ; 0.933]$} & -- & $100.0 \%$ \\
\hline \multicolumn{3}{|c|}{ Heterogeneity: $R=0 \%, \tau^{2}=0, P=.50$} & $50.90 .95 \quad 1$ & & & & \\
\hline & & & Surgery Rate & & & & \\
\hline
\end{tabular}

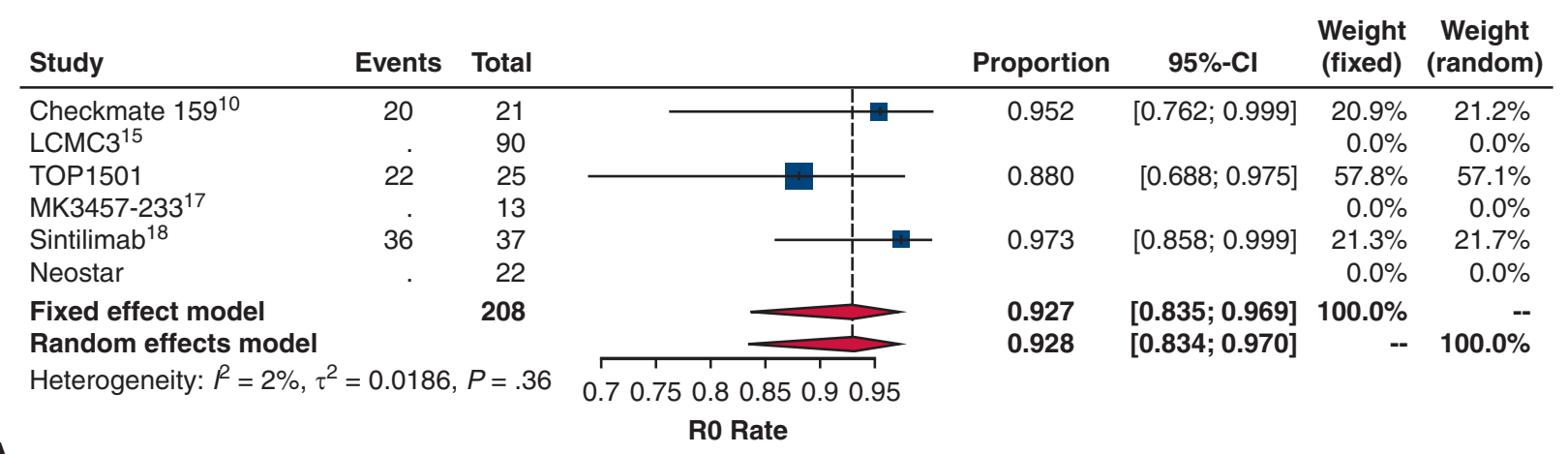

A

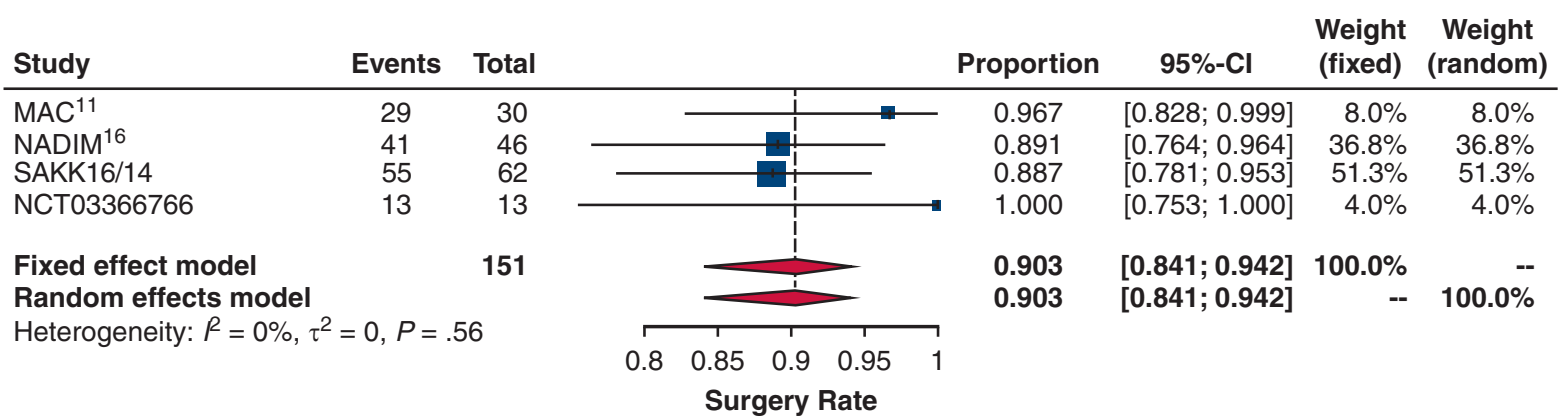

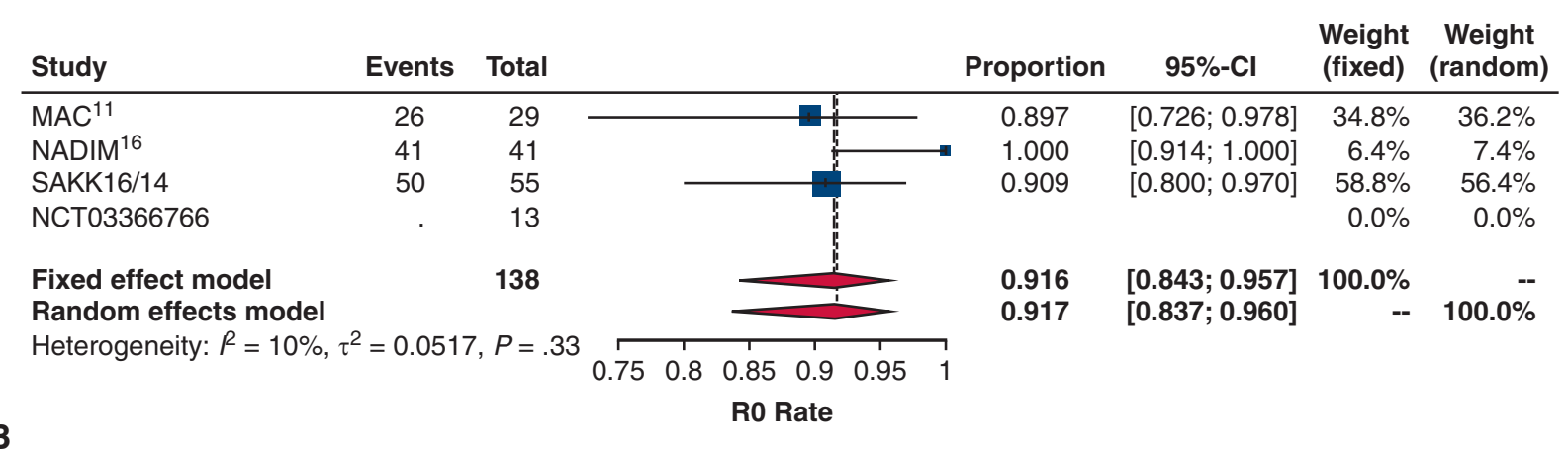

FIGURE 3. Estimates of surgical resection rate and $\mathrm{R} 0$ resection rate in 3 different treatment cohorts. Vertical lines in the forest plots indicates estimates of $\mathrm{R} 0$ resection rate or surgical resection rate in different statistical models. The weights of each individual study in the different statistical models are provided on the right side of forest plots. A, Neoadjuvant immunotherapy (NeoIO) alone. B, NeoIO plus chemotherapy (CT). C, Neoadjuvant CT alone. CI, Confidence interval.

also may be attributed to negative findings in radiologic response owing to short-term drug exposure in immunotherapy. ${ }^{30-33}$ Indeed, the combination strategy of neoadjuvant immunotherapy and chemotherapy was found to have a higher response rate for both radiologic response and pathologic regression. However, very few combination neoadjuvant trials were included in this study, which could have underpowered our ability to 


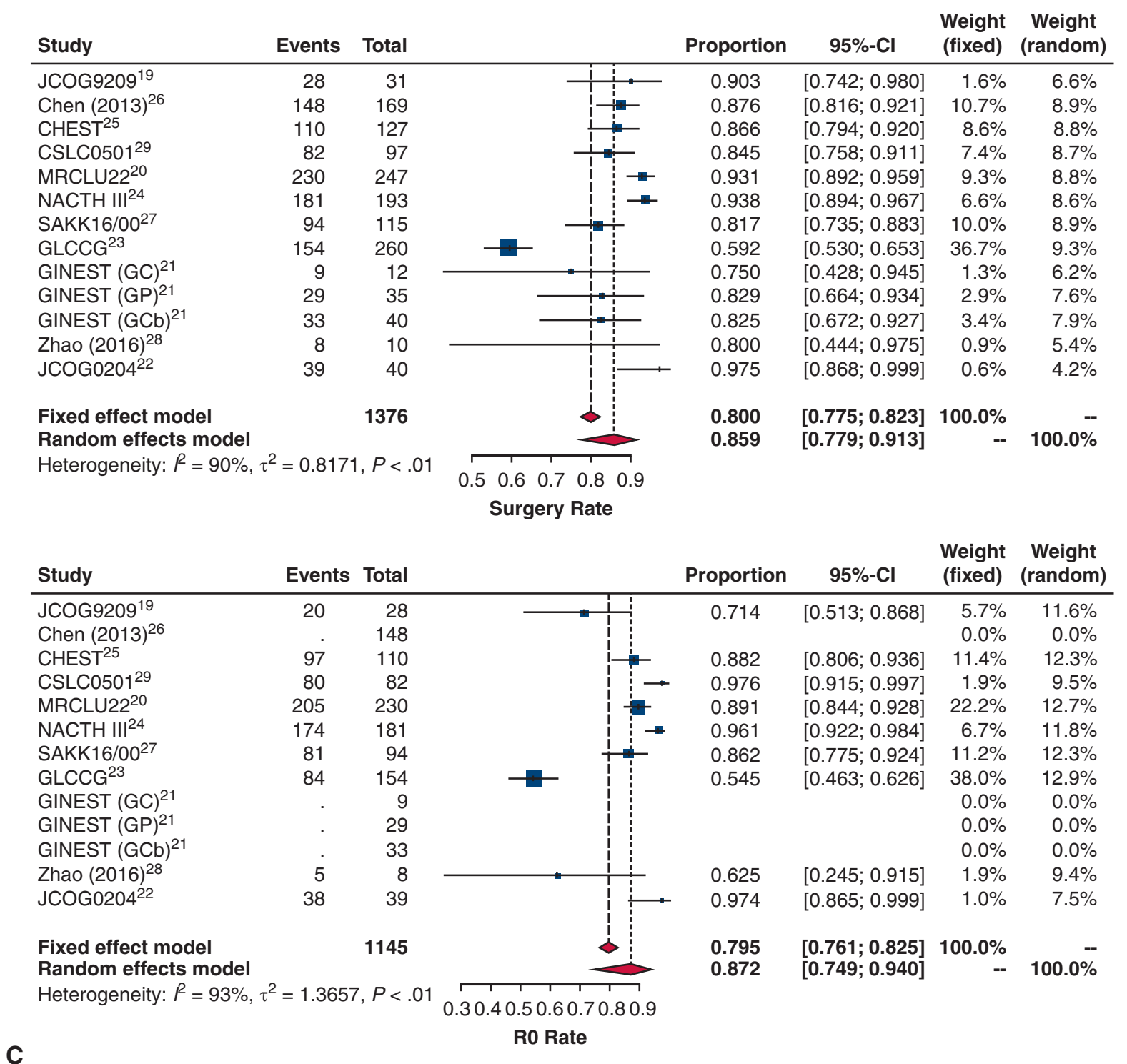

FIGURE 3. Continued.

draw robust conclusions. When we adjusted for the clinical characteristics, we found no notable impact on the overall conclusions. PD-L1, as a well-known biomarker of immunotherapy, ${ }^{34}$ was not eligible for further analysis in this study owing to the limited data on PD-L1 expression in both neoadjuvant immunotherapy and chemotherapy trials. Moreover, although the pivotal role of molecular testing for localized NSCLC is recognized, it remains unclear whether patients who bear a driver mutation, such as $E G F R$, will respond as well to neoadjuvant immunotherapy as patients with advanced cancer, because not all trials involving neoadjuvant immunotherapy excluded $E G F R$ and other oncogene mutations. In several reported neoadjuvant immunotherapy trials, patients with sensitive EGFR mutation achieved MPR or
pCR following neoadjuvant immunotherapy plus chemotherapy. ${ }^{9,35}$ Therefore, whether patients with an oncogene alteration such as EGFR are eligible for immunotherapy for early-stage NSCLC remains an open question to be explored.

It also is unclear whether neoadjuvant immunotherapy can provide a noninferior surgical approach compared with chemotherapy. In this study, we found that neoadjuvant immunotherapy alone or in combination with chemotherapy was associated with a higher surgical rate compared to chemotherapy. Moreover, the R0 resection rate was also higher in the neoadjuvant immunotherapy group. This also was demonstrated in recently released results from Checkmate816 showing elevated surgical and R0 resection rates with neoadjuvant immunotherapy compared with 


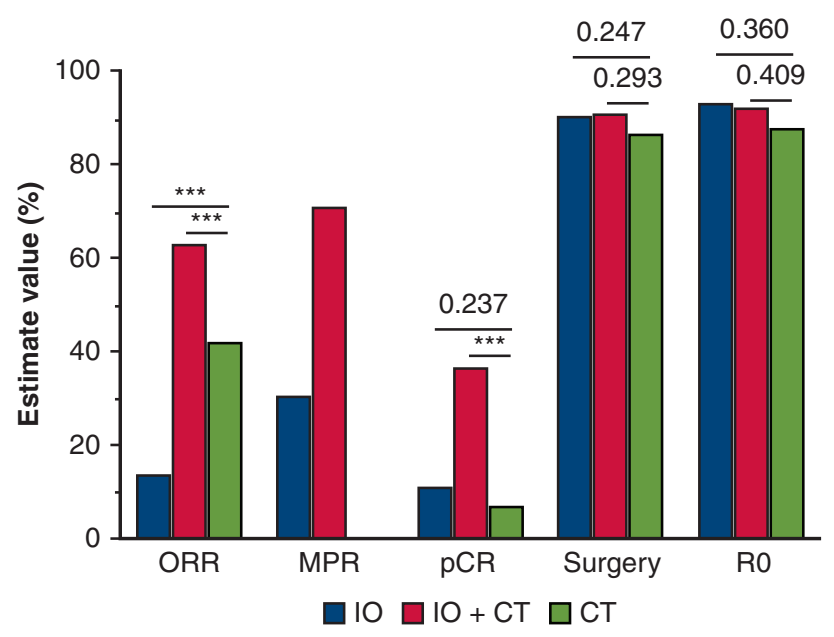

FIGURE 4. Bar graph comparing short-term clinical outcomes with neoadjuvant immunotherapy $(I O)$, IO plus chemotherapy $(C T)$, and CT. Meta-regression was applied to evaluate statistical significance. Because no major pathologic response $(M P R)$ data for neoadjuvant $\mathrm{CT}$ were reported, comparisons were made only with regard to objective response rate $(O R R)$, pathologic complete response $(p C R)$, surgical resection rate, and $\mathrm{R} 0$ resection rate. $* * * P<.001$.

chemotherapy. ${ }^{36}$ Specifically, for patients with stage III NSCLC, we found that neoadjuvant immunotherapy was associated with provide better surgical outcomes compared with neoadjuvant chemotherapy. However, this finding might be confounded by differences in the stages of enrolled patients from these trials.

\section{Study Limitations}

Despite the expected results, this study has several limitations. First, most of the included neoadjuvant immunotherapy trials were single-arm early-phase trials with limited sample sizes and undetermined EGFR mutation status, which could have led to potential bias; therefore, these results should be interpreted with caution. Moreover, some of the trials were not published officially, providing limited clinical information available for the subgroup analysis. Second, the study is a meta-analysis that may have the intrinsic defects of cross-trial comparisons. As the consequence, the statistic power may not be available for consolidate conclusions. Moreover, we did not include trials of neoadjuvant chemotherapy and radiotherapy that showed relatively higher response rates compared with those of chemotherapy alone. Third, the study has potential selection bias. Most of the neoadjuvant chemotherapy trials were published before 2010, whereas the neoadjuvant immunotherapy trials were reported within 5 years. Both efficacy and outcome estimation may be varied owing to uncontrollable factors, such as the standard shift in Response

Study flow chart of pooled analysis for neoadjuvant immunotherapy and chemotherapy

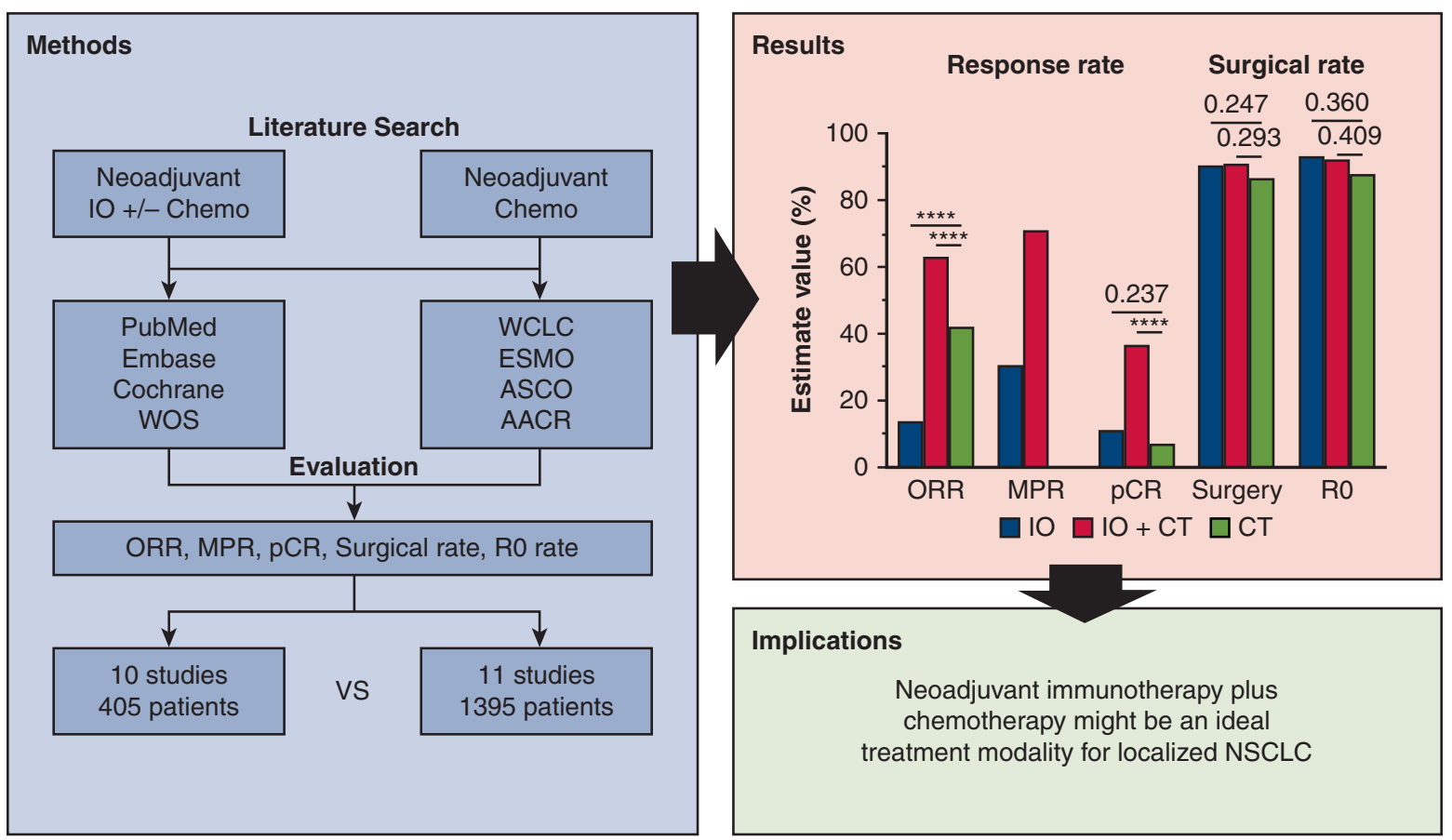

FIGURE 5. Study flow chart. Twenty-one studies were included for pooled analysis to estimate the short-term clinical outcome of neoadjuvant immunotherapy and chemotherapy. $I O$, Immunotherapy; $O R R$, objective response rate; $M P R$, major pathological response; $p C R$, pathological complete response; $C T$, chemotherapy; NSCLC, non-small-cell lung cancer. 
Evaluation Criteria in Solid Tumors (RECIST) and treatment paradigm. In addition, technological advances, including routine use of positron emission tomography/ computed tomography for clinical staging, changes in the staging system, etc, might have affected the proportion of patients being assigned to surgery, as well as the R0 resection rate. Indeed, this is an inevitable bias, given that no phase III neoadjuvant chemotherapy trials have been initiated in decades, owing to the paradigm shift toward neoadjuvant treatment. Finally, results from neoadjuvant immunotherapy trials also may be influenced by the different drugs used, including PD-1 and PD-L1 inhibitors. Although we did not included any randomized trials comparing the efficacy of PD-1 and PD-L1 inhibitors, a previous meta-analysis showed that PD-1 inhibitors were associated with more favorable survival outcomes compared with PD-L1 inhibitors in advanced cancer. ${ }^{37}$ In the present study, we observed a similar trend, with more favorable short-term clinical outcomes associated with PD-1 inhibitors compared with chemotherapy; however, this finding should be interpreted with caution, given the limited number of trials involving PD-L1 inhibitors included.

\section{CONCLUSIONS}

This integrated meta-analysis of high-quality prospective phase II trials indicates that neoadjuvant immunotherapy can provide a deeper pathologic regression along with improved surgical outcomes, especially for patients with stage III NSCLC. Specifically, immunotherapy combined with chemotherapy could be a more ideal treatment modality when clinically available. Recently released data from the phase III Checkmate816 study further support the use of neoadjuvant immunotherapy plus chemotherapy, although long-term outcome data are still pending. In addition, other ongoing phase III trials, such as AEGEAN and IMpower030, are also attempting to illustrate the clinical efficacy of neoadjuvant immunotherapy plus chemotherapy, and other trials, including NEOMUN and NeoCOAST, are exploring the use of neoadjuvant immunotherapy combined with concurrent radiotherapy or other novel agents. These trials will provide further high-level evidence for neoadjuvant treatment and long-term results, helping guide clinical practice.

\section{Conflict of Interest Statement}

W.-Z.Z. has received honoraria from AstraZeneca and Roche. Y.-L.W. has received honoraria from AstraZeneca, Eli Lilly, Roche, Pierre Fabre, Pfizer, and Sanofi and has served in a consulting or advisory role with AstraZeneca, Roche, Merck, and Boehringer Ingelheim and Roche. All other authors have no conflicts of interest to disclose.

The Journal policy requires editors and reviewers to disclose conflicts of interest and to decline handling or reviewing manuscripts for which they may have a conflict of interest. The editors and reviewers of this article have no conflicts of interest.

We thank Jin-Hui Fan for assistance with data collection and Yu Wang and Shan-Mei Liao for statistical analysis consultation. We also thank Yue Li and Zhi-meng Xu for assistance with the systematic literature search according to the study objective predefined by Chao Zhang. We also thank Editage for providing the language editing.

\section{References}

1. Hirsch FR, Scagliotti GV, Mulshine JL, Kwon R, Curran WJ Jr, Wu YL, et al. Lung cancer: current therapies and new targeted treatments. Lancet. 2017;389: 299-311.

2. Wright G, Manser RL, Byrnes G, Hart D, Campbell DA. Surgery for non-small cell lung cancer: systematic review and meta-analysis of randomised controlled trials. Thorax. 2006;61:597-603.

3. Arriagada R, Bergman B, Dunant A, Le Chevalier T, Pignon JP, Vansteenkiste J. Cisplatin-based adjuvant chemotherapy in patients with completely resected non-small-cell lung cancer. N Engl J Med. 2004;350:351-60.

4. NSCLC Meta-analysis Collaborative Group. Preoperative chemotherapy for non-small-cell lung cancer: a systematic review and meta-analysis of individual participant data. Lancet. 2014;383:1561-71.

5. Früh M, Rolland E, Pignon JP, Seymour L, Ding K, Tribodet H, et al. Pooled analysis of the effect of age on adjuvant cisplatin-based chemotherapy for completely resected non-small-cell lung cancer. J Clin Oncol. 2008;26:3573-81.

6. Pignon JP, Tribodet H, Scagliotti GV, Douillard JY, Shepherd FA, Stephens RJ, et al. Lung adjuvant cisplatin evaluation: a pooled analysis by the LACE collaborative group. J Clin Oncol. 2008;26:3552-9.

7. Strauss GM, Herndon JE II, Maddaus MA, Johnstone DW, Johnson EA, Harpole DH, et al. Adjuvant paclitaxel plus carboplatin compared with observation in stage IB non-small-cell lung cancer: CALGB 9633 with the Cancer and Leukemia Group B, Radiation Therapy Oncology Group, and North Central Cancer Treatment Group study groups. J Clin Oncol. 2008;26:5043-51.

8. Forde PM, Chaft JE, Smith KN, Anagnostou V, Cottrell TR, Hellmann MD, et al. Neoadjuvant PD-1 blockade in resectable lung cancer. N Engl J Med. 2018;378: 1976-86.

9. Shu CA, Gainor JF, Awad MM, Chiuzan C, Grigg CM, Pabani A, et al. Neoadjuvant atezolizumab and chemotherapy in patients with resectable non-smallcell lung cancer: an open-label, multicentre, single-arm, phase 2 trial. Lancet Oncol. 2020;21:786-95.

10. William WN Jr, Pataer A, Kalhor N, Correa AM, Rice DC, Wistuba II, et al. Computed tomography RECIST assessment of histopathologic response and prediction of survival in patients with resectable non-small-cell lung cancer after neoadjuvant chemotherapy. J Thorac Oncol. 2013;8:222-8.

11. DerSimonian R, Laird N. Meta-analysis in clinical trials revisited. Contemp Clin Trials. 2015;45(Pt A):139-45.

12. Egger M, Davey Smith G, Schneider M, Minder C. Bias in meta-analysis detected by a simple, graphical test. BMJ. 1997;315:629-34.

13. Oezkan F, He K, Owen DH, Pietrzak M, Cho JH, Kitzler R, et al. OA13.07 neoadjuvant atezolizumab in resectable NSCLC patients: immunophenotyping results from the interim analysis of the multicenter trial LCMC3. J Thorac Oncol. 2019;14:S242-3.

14. Provencio M, Nadal E, Insa A, Garcia Campelo R, Casal J, Domine M, et al. OA13.05 NADIM study: updated clinical research and outcomes. J Thorac Oncol. 2019;14:S241.

15. Ready N, Tong B, Clarke J, Gu L, Wigle D, Dragnev K, et al. P2.04-89 neoadjuvant pembrolizumab in early stage non-small cell lung cancer (NSCLC): toxicity, efficacy, and surgical outcomes. J Thorac Oncol. 2019;14:S745.

16. Gao S, Li N, Gao S, Xue Q, Ying J, Wang S, et al. Neoadjuvant PD-1 inhibitor (Sintilimab) in NSCLC. J Thorac Oncol. 2020;15:816-26.

17. Nagai K, Tsuchiya R, Mori T, Tada H, Ichinose Y, Koike T, et al. A randomized trial comparing induction chemotherapy followed by surgery with surgery alone for patients with stage IIIA N2 non-small cell lung cancer (JCOG 9209). J Thorac Cardiovasc Surg. 2003;125:254-60.

18. Gilligan D, Nicolson M, Smith I, Groen H, Dalesio O, Goldstraw P, et al. Preoperative chemotherapy in patients with resectable non-small cell lung cancer: results of the MRC LU22/NVALT 2/EORTC 08012 multicentre randomised trial and update of systematic review. Lancet. 2007;369:1929-37. 
19. Detterbeck FC, Socinski MA, Gralla RJ, Edelman MJ, Jahan TM, Loesch DM, et al. Neoadjuvant chemotherapy with gemcitabine-containing regimens in patients with early-stage non-small cell lung cancer. J Thorac Oncol. 2008;3:37-45.

20. Kunitoh H, Kato H, Tsuboi M, Asamura H, Tada H, Naga K, et al. A randomised phase II trial of preoperative chemotherapy of cisplatin-docetaxel or docetaxel alone for clinical stage IB/II non-small-cell lung cancer results of a Japan Clinical Oncology Group trial (JCOG 0204). Br J Cancer. 2008;99:852-7.

21. Thomas M, Rübe C, Hoffknecht P, Macha HN, Freitag L, Linder A, et al. Effect of preoperative chemoradiation in addition to preoperative chemotherapy: a randomised trial in stage III non-small-cell lung cancer. Lancet Oncol. 2008;9: 636-48.

22. Felip E, Rosell R, Maestre JA, Rodríguez-Paniagua JM, Morán T, Astudillo J, et al. Preoperative chemotherapy plus surgery versus surgery plus adjuvant chemotherapy versus surgery alone in early-stage non-small-cell lung cancer. J Clin Oncol. 2010;28:3138-45.

23. Scagliotti GV, Pastorino U, Vansteenkiste JF, Spaggiari L, Facciolo F, Orlowski TM, et al. Randomized phase III study of surgery alone or surgery plus preoperative cisplatin and gemcitabine in stages IB to IIIA non-small-cell lung cancer. J Clin Oncol. 2012;30:172-8.

24. Chen Z, Luo Q, Jian H, Zhou Z, Cheng B, Lu S, et al. Long-term results of a randomized controlled trial evaluating preoperative chemotherapy in resectable non-small cell lung cancer. Onco Targets Ther. 2013;6:645-50.

25. Pless M, Stupp R, Ris HB, Stahel RA, Weder W, Thierstein S, et al. Induction chemoradiation in stage IIIA/N2 non-small-cell lung cancer: a phase 3 randomised trial. Lancet. 2015;386:1049-56.

26. Zhao X, Su Y, You J, Gong L, Zhang Z, Wang M, et al. Combining antiangiogenic therapy with neoadjuvant chemotherapy increases treatment efficacy in stage IIIA (N2) non-small cell lung cancer without increasing adverse effects. Oncotarget. 2016;7:62619-26.

27. Yang XN, Zhong WZ, Ben XS, Luo HH, Wang C, Wang Q, et al. OA09.03 randomized controlled study comparing adjuvant versus neo-adjuvant chemotherapy in resectable stage IB to IIIA NSCLC. J Thorac Oncol. 2017;12:S277-8.

28. Chemi F, Rothwell DG, McGranahan N, Gulati S, Abbosh C, Pearce SP, et al. Pulmonary venous circulating tumor cell dissemination before tumor resection and disease relapse. Nat Med. 2019;25:1534-9.
29. Nagasaka M, Gadgeel SM. Role of chemotherapy and targeted therapy in early-stage non-small cell lung cancer. Expert Rev Anticancer Ther. 2018; 18:63-70.

30. Borghaei H, Paz-Ares L, Horn L, Spigel DR, Steins M, Ready NE, et al. Nivolumab versus docetaxel in advanced nonsquamous non-small-cell lung cancer. $N$ Engl J Med. 2015;373:1627-39.

31. Brahmer J, Reckamp KL, Baas P, Crinò L, Eberhardt WEE, Poddubskaya E, et al. Nivolumab versus docetaxel in advanced squamous-cell non-small-cell lung cancer. N Engl J Med. 2015;373:123-35.

32. Herbst RS, Baas P, Kim DW, Felip E, Pérez-Gracia JL, Han JY, et al. Pembrolizumab versus docetaxel for previously treated, PD-L1-positive, advanced nonsmall-cell lung cancer (KEYNOTE-010): a randomised controlled trial. Lancet 2016;387:1540-50.

33. Reck M, Rodríguez-Abreu D, Robinson AG, Hui R, Csőszi T, Fülöp A, et al Pembrolizumab versus chemotherapy for PD-L1-positive non-small-cell lung cancer. N Engl J Med. 2016;375:1823-33.

34. Diggs LP, Hsueh EC. Utility of PD-L1 immunohistochemistry assays for predicting PD-1/PD-L1 inhibitor response. Biomark Res. 2017;5:12.

35. Provencio M, Nadal E, Insa A, García-Campelo MR, Casal-Rubio J, Dómine M, et al. Neoadjuvant chemotherapy and nivolumab in resectable non-small-cell lung cancer (NADIM): an open-label, multicentre, single-arm, phase 2 trial. Lancet Oncol. 2020;21:1413-22.

36. Spicer J. Surgical outcomes from the phase 3 CheckMate 816 trial: nivolumab (NIVO) + platinum-doublet chemotherapy (chemo) vs chemo alone as neoadjuvant treatment for patients with resectable non-small cell lung cancer (NSCLC). J Clin Oncol. 2021;39(Suppl 15):8503.

37. Duan J, Cui L, Zhao X, Bai H, Cai S, Wang G, et al. Use of immunotherapy with programmed cell death 1 vs programmed cell death ligand 1 inhibitors in patients with cancer: a systematic review and meta-analysis. JAMA Oncol. 2020;6: $375-84$.

Key Words: neoadjuvant immunotherapy, non-small cell lung cancer, meta-analysis, pathologic complete response, major pathologic response 


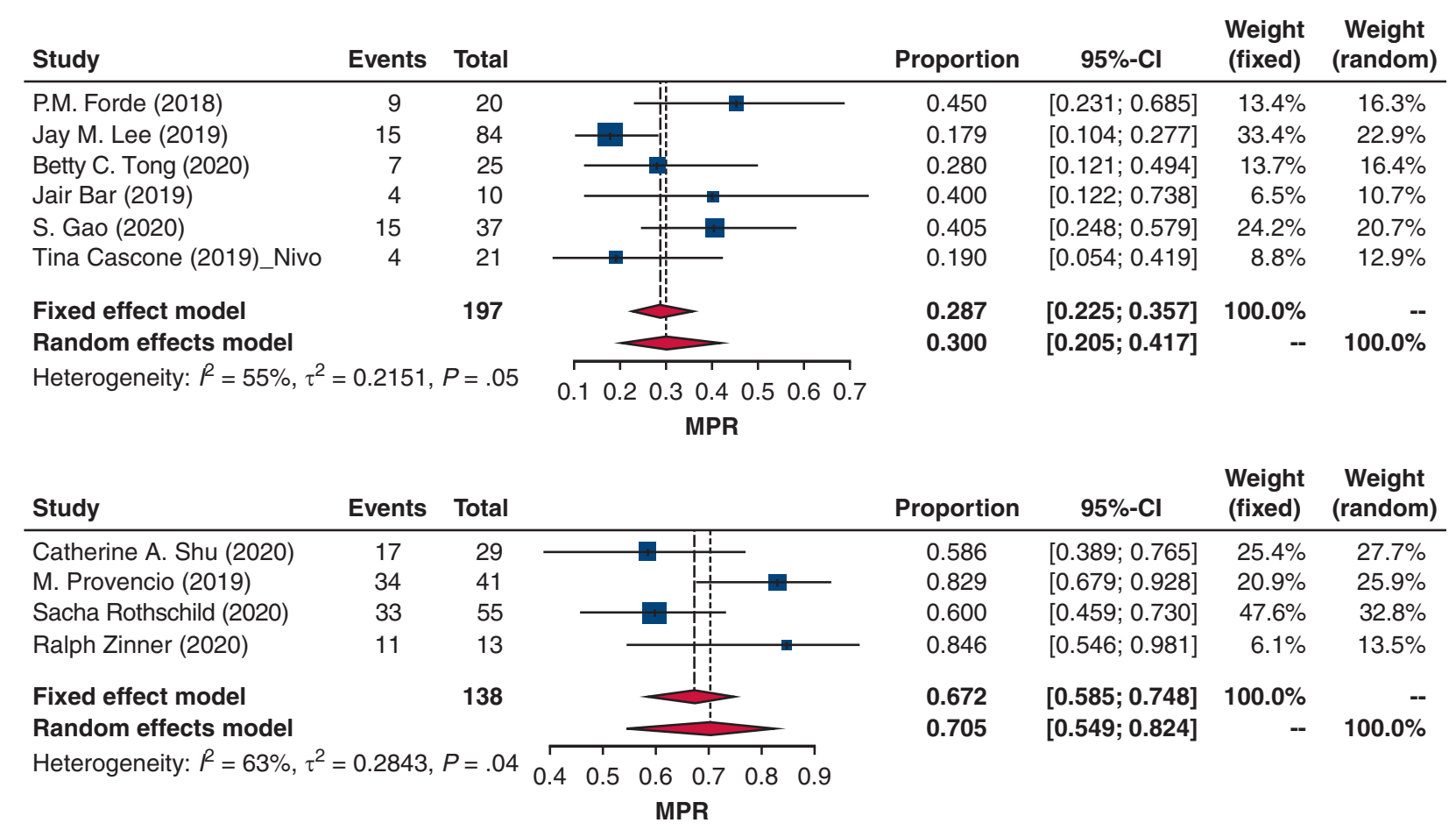

FIGURE E1. Estimation ratio of major pathologic response $(M P R)$ between neoadjuvant immunotherapy alone and combination immunotherapy. $C I$, Confidence interval. 

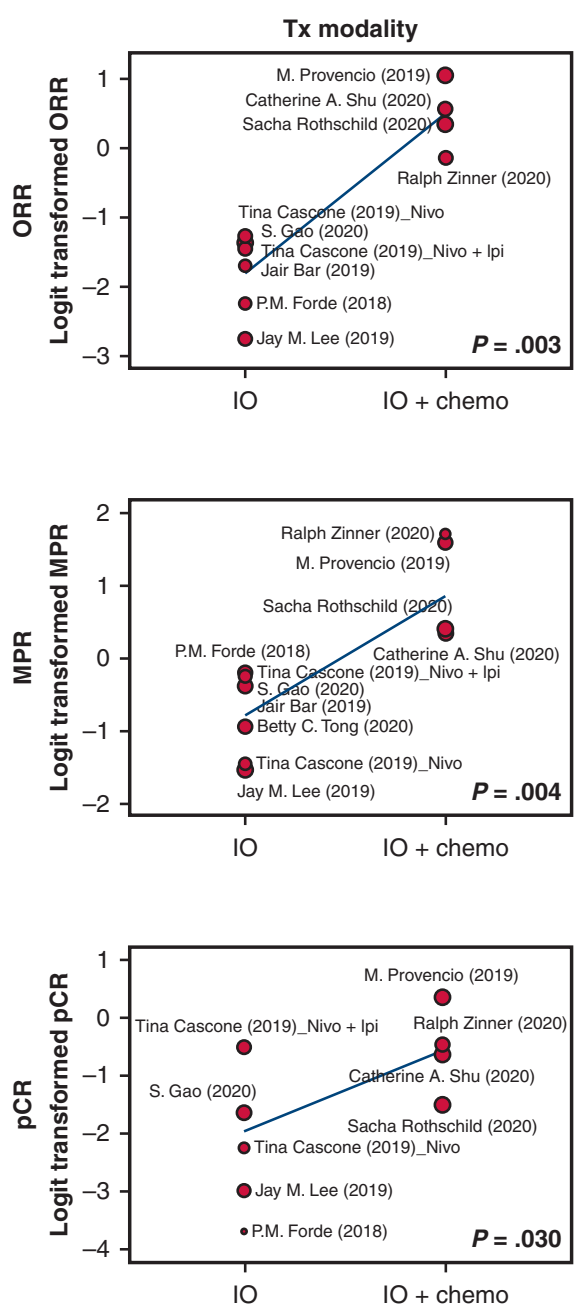
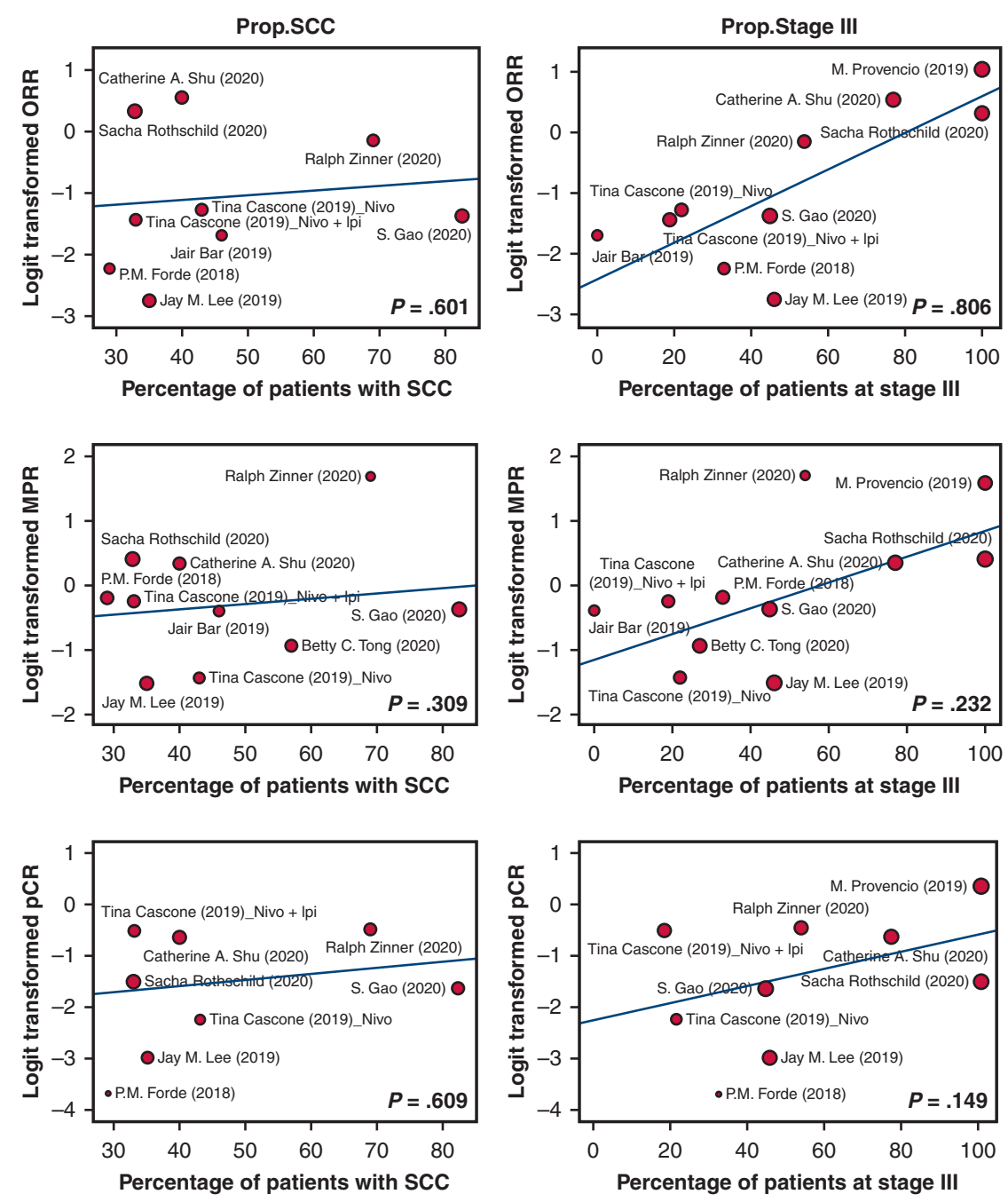

FIGURE E2. Meta-regression on the proportion of squamous cell carcinoma and stage III disease among different clinical outcomes for neoadjuvant immunotherapy. Tx, Treatment; ORR, objective response rate; Prop, proportion; SCC, squamous cell carcinoma; $M P R$, major pathologic response; $p C R$, pathologic complete response.
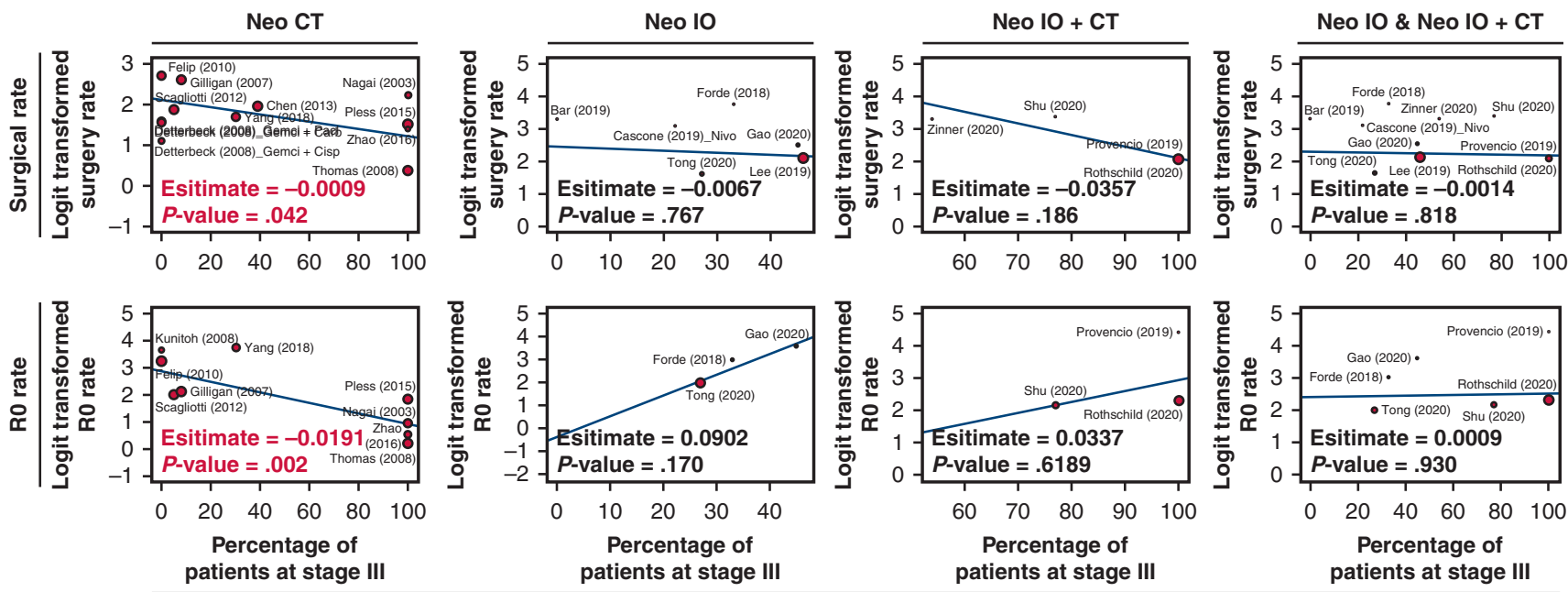

Proportion of Stage III NSCLC

FIGURE E3. Meta-regression on surgical feasibility between neoadjuvant immunotherapy $(\mathrm{NeoIO})$ and chemotherapy $(\mathrm{NeoCT})$ regarding the proportion of stage III patients. NSCLC, Non-small cell lung cancer. 


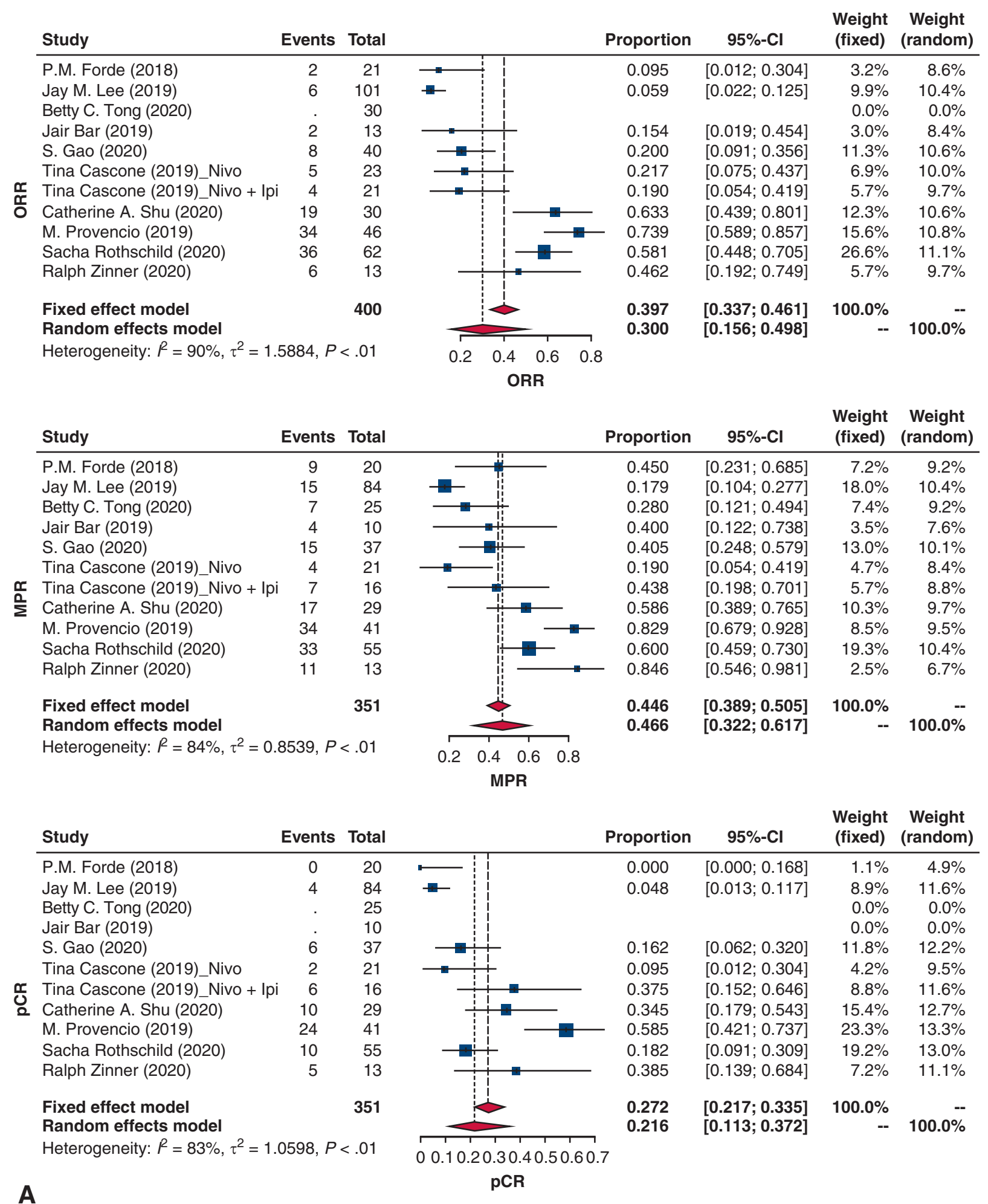

FIGURE E4. Forest plots (A) for Programmed death ligand 1-based single-agent neoadjuvant immunotherapy trials and funnel plots (B) for publication bias estimation. $O R R$, Objective response rate; $C I$, confidence interval; $M P R$, major pathologic response; $p C R$, pathologic complete response. 

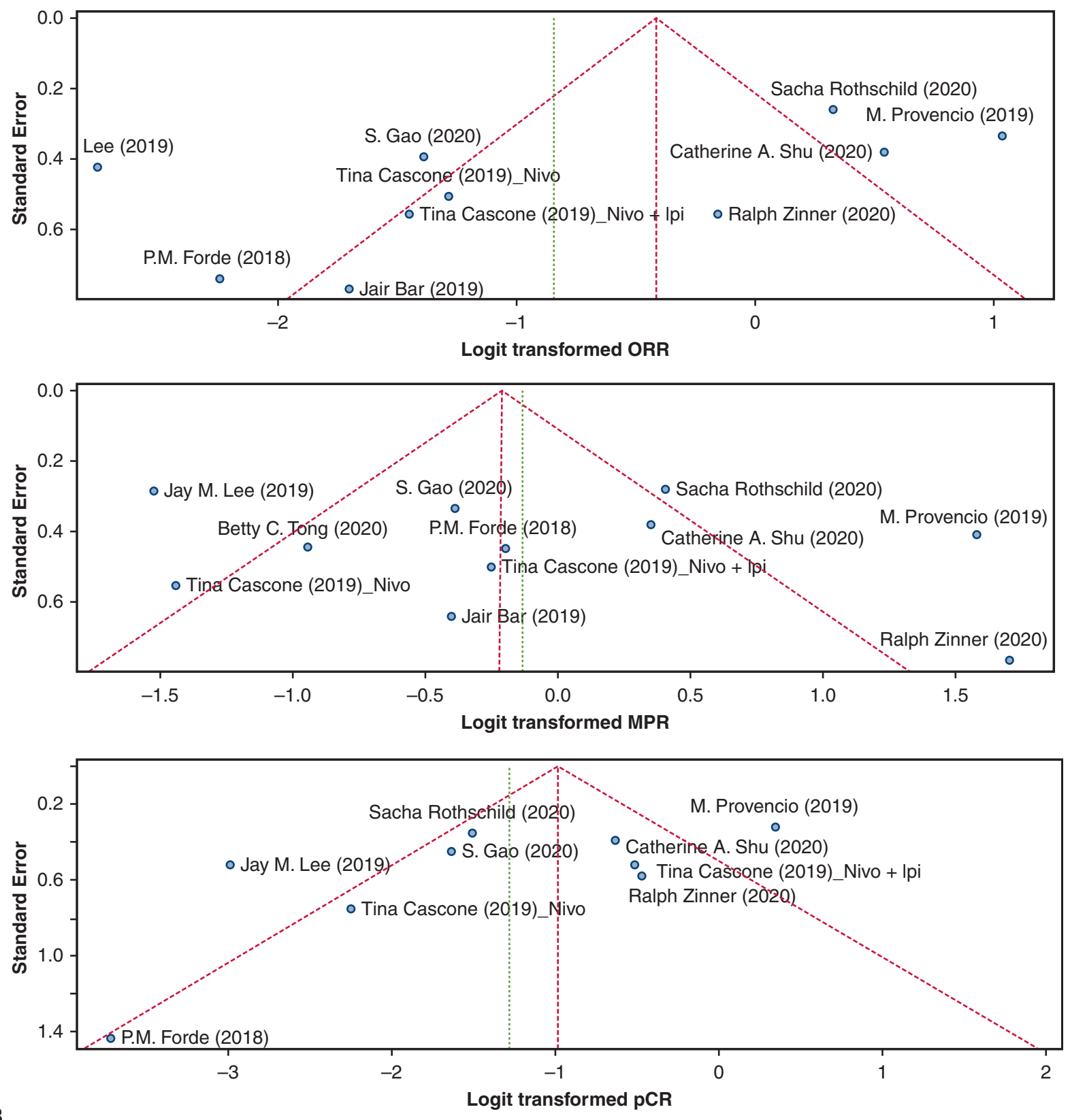

FIGURE E4. Continued. 


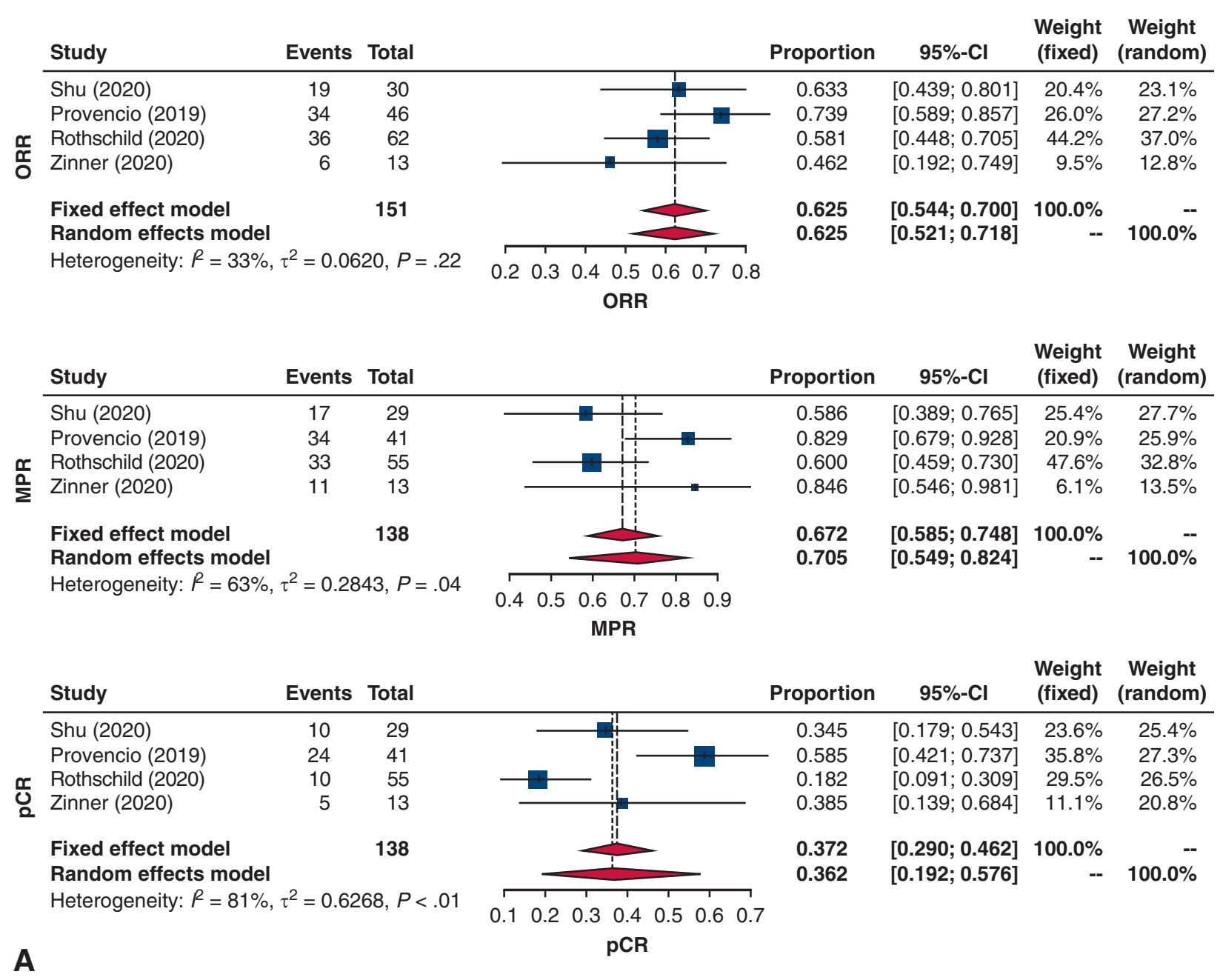

FIGURE E5. Forest plots (A) for neoadjuvant immunotherapy plus chemotherapy trials and funnel plots (B) for publication bias estimation. ORR, Objective response rate; $C I$, confidence interval; $M P R$, major pathologic response; $p C R$, pathologic complete response. 

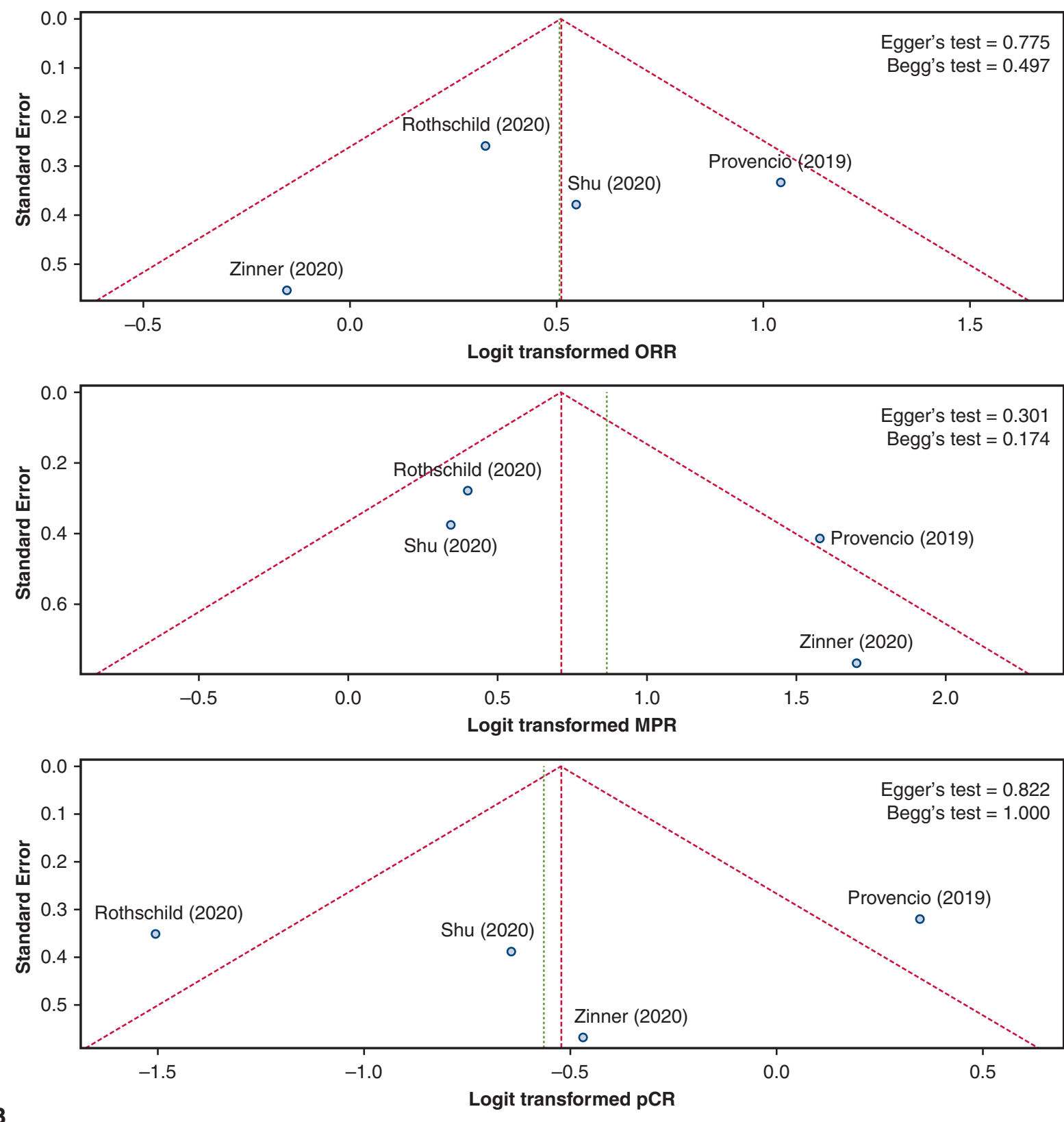

B

FIGURE E5. Continued. 


\begin{tabular}{|c|c|c|c|c|c|c|c|}
\hline Study & Events & Total & & Proportion & $95 \%-\mathrm{Cl}$ & $\begin{array}{l}\text { Weight } \\
\text { (fixed) }\end{array}$ & $\begin{array}{c}\text { Weight } \\
\text { (random) }\end{array}$ \\
\hline Nagai (2003) & 8 & 31 & & 0.258 & {$[0.119 ; 0.446]$} & $1.8 \%$ & $4.4 \%$ \\
\hline Chen (2013) & 79 & 169 & & 0.467 & {$[0.390 ; 0.546]$} & $12.7 \%$ & $10.9 \%$ \\
\hline Scagliotti (2012) & 45 & 127 & & 0.354 & {$[0.272 ; 0.444]$} & $8.8 \%$ & $9.9 \%$ \\
\hline Yang (2018) & 33 & 97 & & 0.340 & {$[0.247 ; 0.443]$} & $6.6 \%$ & $8.9 \%$ \\
\hline Gilligan (2007) & 120 & 247 & & 0.486 & {$[0.422 ; 0.550]$} & $18.7 \%$ & $11.8 \%$ \\
\hline Felip (2010) & 106 & 193 & & 0.549 & {$[0.476 ; 0.621]$} & $14.4 \%$ & $11.3 \%$ \\
\hline Pless (2015) & 50 & 115 & & 0.435 & {$[0.343 ; 0.530]$} & $8.5 \%$ & $9.8 \%$ \\
\hline Thomas (2008) & 120 & 260 & & 0.462 & {$[0.400 ; 0.524]$} & $19.5 \%$ & $11.9 \%$ \\
\hline Detterbeck (2008)_Gemci + Cisp & 5 & 12 & & 0.417 & {$[0.152 ; 0.723]$} & $0.9 \%$ & $2.6 \%$ \\
\hline Detterbeck (2008)_Gemci + Pacl & 12 & 35 & & 0.343 & {$[0.191 ; 0.522]$} & $2.4 \%$ & $5.4 \%$ \\
\hline Detterbeck (2008)_Gemci + Carb & 8 & 40 & & 0.200 & {$[0.091 ; 0.356]$} & $1.9 \%$ & $4.7 \%$ \\
\hline Zhao (2016) & 4 & 10 & & 0.400 & {$[0.122 ; 0.738]$} & $0.7 \%$ & $2.2 \%$ \\
\hline Kunitoh (2008) & 18 & 40 & & 0.450 & {$[0.293 ; 0.615]$} & $3.0 \%$ & $6.1 \%$ \\
\hline Fixed effect model & & 1376 & & 0.445 & {$[0.419 ; 0.472]$} & $100.0 \%$ & -- \\
\hline Random effects model & & & & 0.416 & {$[0.368 ; 0.467]$} & -- & $100.0 \%$ \\
\hline Heterogeneity: $R=64 \%, \tau^{2}=0.075$ & $750, P<$. & & 07 & & & & \\
\hline & & & ORR & & & & \\
\hline Study & Events & Total & & Proportion & $95 \%-\mathrm{Cl}$ & $\begin{array}{l}\text { Weight } \\
\text { (fixed) }\end{array}$ & $\begin{array}{l}\text { Weight } \\
\text { (random) }\end{array}$ \\
\hline Nagai (2003) & 0 & 28 & 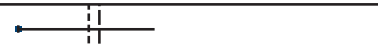 & 0.000 & {$[0.000 ; 0.123]$} & $1.1 \%$ & $2.5 \%$ \\
\hline Scagliotti (2012) & 5 & 110 & $\rightarrow+$ & 0.045 & {$[0.015 ; 0.103]$} & $10.7 \%$ & $14.5 \%$ \\
\hline Gilligan (2007) & 8 & 224 & & 0.036 & {$[0.016 ; 0.069]$} & $17.4 \%$ & $18.3 \%$ \\
\hline Felip (2010) & 19 & 181 & & 0.105 & {$[0.064 ; 0.159]$} & $38.3 \%$ & $24.0 \%$ \\
\hline Pless (2015) & 11 & 94 & & 0.117 & {$[0.060 ; 0.200]$} & $21.9 \%$ & $20.1 \%$ \\
\hline Thomas (2008) & . & 84 & & & & $0.0 \%$ & $0.0 \%$ \\
\hline Detterbeck (2008)_Gemci + Cisp & 0 & 9 & & 0.000 & {$[0.000 ; 0.336]$} & $1.1 \%$ & $2.4 \%$ \\
\hline Detterbeck (2008)_Gemci + Pacl & 2 & 29 & & 0.069 & {$[0.008 ; 0.228]$} & $4.2 \%$ & $7.8 \%$ \\
\hline Detterbeck (2008)_Gemci + Carb & 0 & 33 & & 0.000 & {$[0.000 ; 0.106]$} & $1.1 \%$ & $2.5 \%$ \\
\hline Zhao (2016) & . & 8 & & & & $0.0 \%$ & $0.0 \%$ \\
\hline Kunitoh (2008) & 2 & 39 & & 0.051 & {$[0.006 ; 0.173]$} & $4.3 \%$ & $7.9 \%$ \\
\hline Fixed effect model & & 839 & & 0.075 & {$[0.057 ; 0.098]$} & $100.0 \%$ & -- \\
\hline Random effects model & & & & 0.064 & {$[0.042 ; 0.098]$} & -- & $100.0 \%$ \\
\hline Heterogeneity: $R=42 \%, \tau^{2}=0.169$ & 396, $P=$ & & & & & & \\
\hline & & & $0 \quad 0.050 .10 .15$ & & & & \\
\hline & & & pCR & & & & \\
\hline
\end{tabular}

FIGURE E6. Forest plots (A) for neoadjuvant chemotherapy trials and funnel plots (B) for publication bias estimation. ORR, Objective response rate; $C I$, confidence interval; $p C R$, pathologic complete response. 

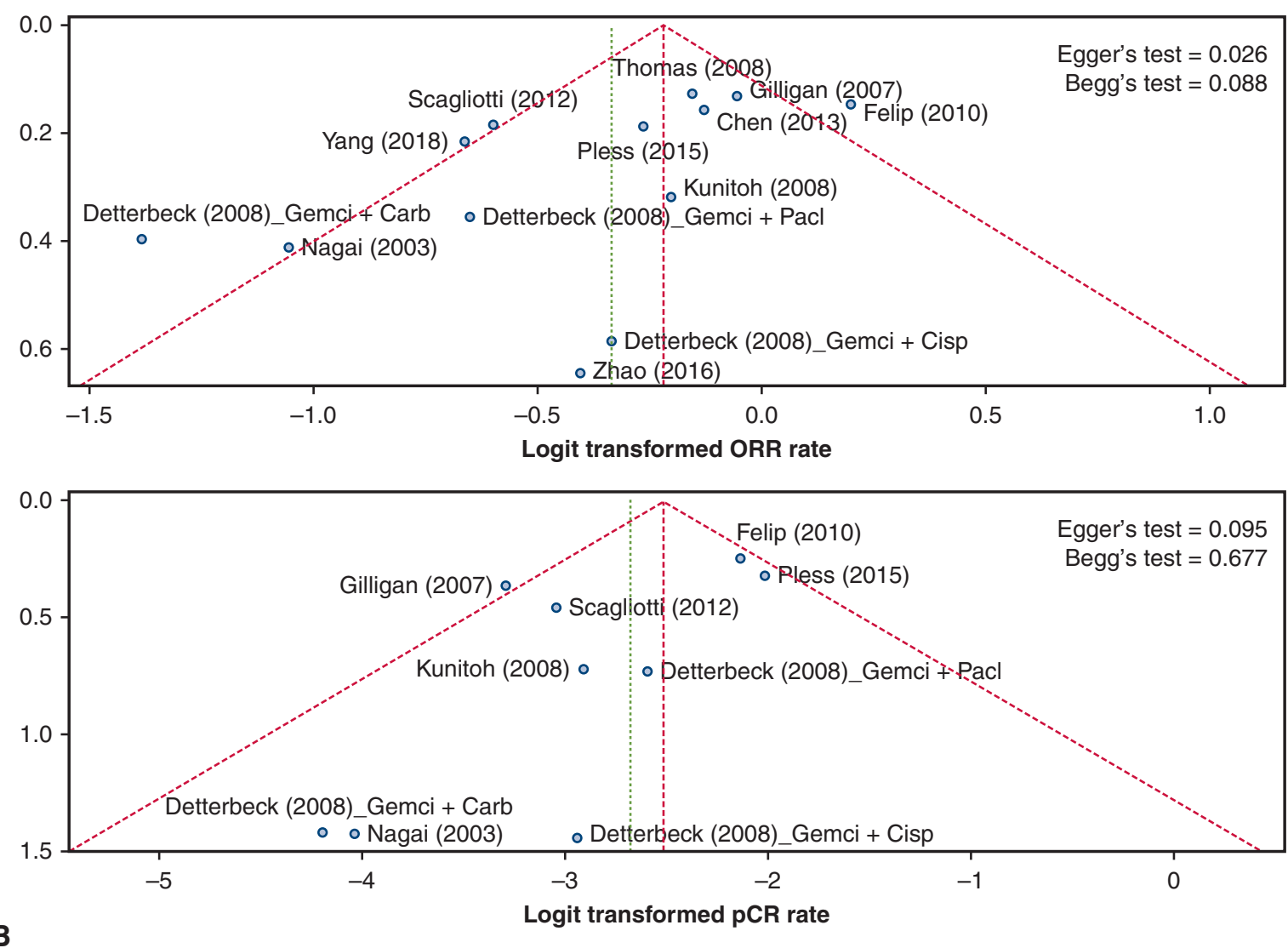

FIGURE E6. Continued.

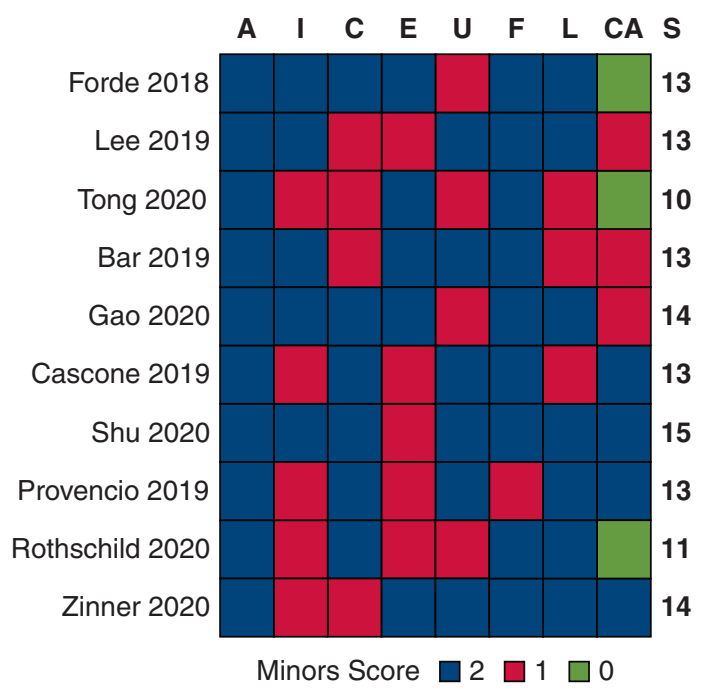

A

FIGURE E7. Risk of bias assessment for neoadjuvant immunotherapy and chemotherapy trials. In A: A, a clearly stated aim; I, inclusion of consecutive patients; C, prospective collection of data; E, Endpoint appropriate to the aim of study; U, unbiased assessment of the study endpoint; F, Follow-up period appropriate to the aim of the study; L, loss to follow-up $<5 \%$; CA, prospective calculation of the study size; R, random sequence generation. In B: A, allocation concealment; BP, blinding of participants and personnel; BA, blinding of outcome assessment; I, incomplete outcome data; S, selective reporting; O, other bias.

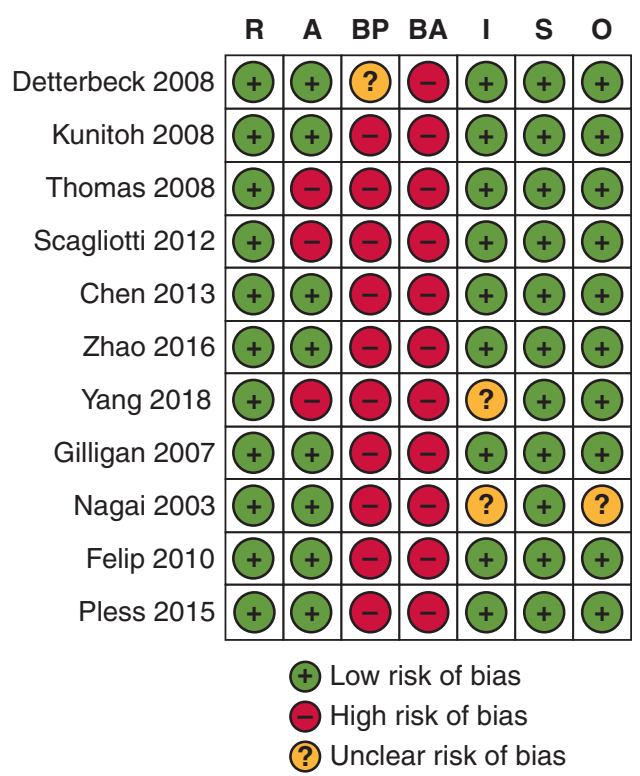

B 